TRANSACTIONS OF THE

AMERICAN MATHEMATICAL SOCIETY

Volume 358, Number 10, October 2006, Pages 4605-4635

S 0002-9947(06)03900-6

Article electronically published on May 9, 2006

\title{
FINITE EDGE-TRANSITIVE CAYLEY GRAPHS AND ROTARY CAYLEY MAPS
}

\author{
CAI HENG LI
}

\begin{abstract}
This paper aims to develop a theory for studying Cayley graphs, especially for those with a high degree of symmetry. The theory consists of analysing several types of basic Cayley graphs (normal, bi-normal, and corefree), and analysing several operations of Cayley graphs (core quotient, normal quotient, and imprimitive quotient). It provides methods for constructing and characterising various combinatorial objects, such as half-transitive graphs, (orientable and non-orientable) regular Cayley maps, vertex-transitive nonCayley graphs, and permutation groups containing certain regular subgroups.

In particular, a characterisation is given of locally primitive holomorph Cayley graphs, and a classification is given of rotary Cayley maps of simple groups. Also a complete classification is given of primitive permutation groups that contain a regular dihedral subgroup.
\end{abstract}

\section{INTRODUCTION}

A digraph $\Gamma$ is a Cayley digraph if there exist a group $G$ and a subset $S \subset G$ such that the vertices of $\Gamma$ may be identified with the elements of $G$ in the way that $x$ is connected to $y$ if and only if $y x^{-1} \in S$. The Cayley digraph $\Gamma$ is denoted by Cay $(G, S)$, and the group $G$ is sometimes called a base group of $\Gamma$. If for each element $s \in S$, the inverse $s^{-1}$ also lies in $S$, then the adjacency is symmetric and thus Cay $(G, S)$ may be viewed as an (undirected) graph, that is, a Cayley graph.

A Cayley digraph $\Gamma=\operatorname{Cay}(G, S)$ has an automorphism group

$$
\hat{G}=\{\hat{g}: x \rightarrow x g \text { for all } x \in G \mid g \in G\},
$$

consisting of right multiplications of elements $g \in G$. The subgroup $\hat{G}$ acts regularly on the vertex set of $\Gamma$, and in particular, $\Gamma$ is vertex-transitive. It is actually easily shown that a digraph is a Cayley digraph of a group $G$ if and only if its automorphism group contains a subgroup which is isomorphic to $G$ and acts regularly on vertices; see for example [2, Proposition 16.3].

Cayley digraphs stem from a type of diagram now called a Cayley color diagram, which was introduced by A. Cayley in 1878 as a graphical representation of abstract groups. Cayley digraphs have been extensively used in various other areas, such as extremal graph theory and communication networks. This paper aims at developing a theory of Cayley graphs and digraphs, and applying it to the study of other (mainly combinatorial) objects.

Received by the editors April 13, 2004 and, in revised form, October 14, 2004.

2000 Mathematics Subject Classification. Primary 20B15, 20B30, 05C25.

Part of this work was done while the author held a QEII Fellowship from the Australian Research Council. The author is grateful to the referee for constructive suggestions. 
Our theory is built on analysing several special classes of Cayley graphs (defined in Subsection 1.1), and analysing some operations on general Cayley graphs (discussed in Subsection 1.2).

1.1. Basic edge-transitive Cayley graphs. For two groups $X$ and $Y$, denote by $X \rtimes Y$ a semidirect product of $X$ by $Y$, and by $X \circ Y$ the central product of $X$ and $Y$. For a subgroup $G$ of $X$, denote by $\mathbf{N}_{X}(G)$ and $\mathbf{C}_{X}(G)$ the normalizer and the centralizer of $G$ in $X$, respectively.

Let $G$ be a group, and let $\Gamma=\operatorname{Cay}(G, S)$. Denote by 1 the vertex of $\Gamma$ corresponding the identity of $G$. Let

$$
\begin{aligned}
& \text { Aut }(G, S)=\left\{\sigma \in \operatorname{Aut}(G) \mid S^{\sigma}=S\right\}, \\
& \operatorname{lnn}(G, S)=\left\{\sigma \in \operatorname{Inn}(G) \mid S^{\sigma}=S\right\} .
\end{aligned}
$$

Then

$$
\begin{aligned}
& \operatorname{Inn}(G, S) \unlhd \operatorname{Aut}(G, S) \leq(\operatorname{Aut} \Gamma)_{1}=\left\{\tau \in \operatorname{Aut} \Gamma \mid \mathbf{1}^{\tau}=\mathbf{1}\right\}, \\
& \hat{G} \unlhd \hat{G} \rtimes \operatorname{Inn}(G, S) \unlhd \hat{G} \rtimes \operatorname{Aut}(G, S) \leq \operatorname{Aut} \Gamma .
\end{aligned}
$$

The symmetric group $\operatorname{Sym}(G)$ contains another regular subgroup,

$$
\tilde{G}=\left\{\tilde{g}: x \rightarrow g^{-1} x \text { for all } x \in G \mid g \in G\right\},
$$

consisting of left multiplications of elements $g \in G$. However, the group $\tilde{G}$ is not necessarily a subgroup of Aut $\Gamma$. It is known that $\mathbf{N}_{\text {Aut } \Gamma}(\hat{G})=\hat{G} \rtimes \operatorname{Aut}(G, S)$ and

$$
\hat{G} \mathbf{C}_{\text {Aut } \Gamma}(\hat{G})=\hat{G} \circ \tilde{H}=\hat{G} \rtimes \operatorname{lnn}(G, S),
$$

where $\tilde{H}=\{\tilde{h} \in \tilde{G} \mid h \in H\}$; see Lemma 2.1.

The subgroup $\operatorname{Aut}(G, S)$ plays an important role in the study of Cayley graphs; see for example [13, 32, 37. Assume that $\hat{G}<X \leq$ Aut $\Gamma$. Then, the following special classes of Cayley graphs are the basic objects of our study of Cayley graphs:

(a) If $\hat{G}$ is normal in $X$, then $X_{1} \leq \operatorname{Aut}(G, S)$, and $\Gamma$ is called an $X$-normal Cayley graph of $G$. Further, if $\hat{G}$ is a normal subgroup of the full automorphism group Aut $\Gamma$, then Aut $\Gamma=\hat{G} \rtimes \operatorname{Aut}(G, S)$, and $\Gamma$ is called a normal Cayley graph. A special type of normal Cayley graph satisfies $X_{1} \leq \operatorname{lnn}(G, S)$, or equivalently, $\hat{G} \triangleleft X \leq \hat{G} \circ \tilde{G}$. For convenience, in this case, $\Gamma$ is called an $X$-central Cayley graph of $G$. If $X_{\mathbf{1}} \geq \operatorname{Inn}(G)$, then $\operatorname{Cay}(G, S)$ is called a holomorph.

(b) If $\hat{G}$ is not normal in $X$ but has a subgroup of index 2 which is normal in $X$, then $\Gamma$ is called an $X$-bi-normal Cayley graph of $G$.

(c) If $\hat{G}$ is core free in $X$ (that is, $\hat{G}$ contains no non-trivial normal subgroup of $X$ ), then $\Gamma$ is called a core-free Cayley graph of $G$.

For cases (a) and (b) in which $\hat{G}$ exhibits normality or near-normality in $X$, the action of $X$ on $\Gamma$ may be described in terms of the base group in a satisfactory way. For the core-free case (c), the property that $X$ has an exact factorization plays a key role:

$$
X=\hat{G} H \text {, with } H \text { core-free and } \hat{G} \cap H=1 \text {, }
$$

where $H=X_{1}$. A core-free Cayley graph $\Gamma$ generally 'should' be described as a coset graph; see Section 3. For core-free Cayley graphs, we introduce their dual Cayley graphs. This enables us to establish a connection between rotary Cayley maps and circulants graphs, and as well as between regular Cayley maps and dihedral Cayley graphs. 
Studying these three classes of Cayley graphs in 22 led to the proof that 3-arctransitive Cayley graphs are rare. Here we further analyse these Cayley graphs, leading to new constructions of various interesting combinatorial objects. Some of them are stated in the two theorems below.

A graph is said to be half-transitive if it is vertex- and edge-transitive but not arctransitive. The problem of constructing and characterizing half-transitive graphs was initiated by Tutte (1965), and is a currently active topic in algebraic graph theory; see [28, 10, 24, for references. For a group $X$, its socle $\operatorname{soc}(X)$ is the product of all minimal normal subgroups of $X$. A primitive permutation group $X$ on $\Omega$ is said to have twisted wreath product type if $\operatorname{soc}(X)$ is a non-abelian minimal normal subgroup and acts regularly on $\Omega$. The following theorem provides a potential method for constructing half-transitive graphs of large valency; see Example 2.11,

Theorem 1.1. Let $X$ be a primitive permutation group of twisted wreath product type, and write $G=\operatorname{soc}(X)=T_{1} \times \cdots \times T_{l}$ for some $l \geq 5$ so that $X=G \rtimes X_{1}$. Assume that $t$ is an element of $T_{1}$ which is not conjugate in Aut $\left(T_{1}\right)$ to $t^{-1}$. Let $S=\left\{h^{-1} t h, h^{-1} t^{-1} h \mid h \in X_{1}\right\}$. Then, Cay $(G, S)$ is a half-transitive graph.

A group $G$ is called a $B$-group (after Burnside) if each primitive permutation group containing a regular subgroup isomorphic to $G$ is necessarily 2 -transitive. In his famous book 36, H. Wielandt proposed the following question (see 36, p. 69]): If $G$ is a B-group and $H$ is a group such that $|H|$ and $|G|$ are coprime, is $G \times H$ necessarily a B-group? The next theorem gives an infinite family of counterexamples.

Theorem 1.2. Let $q=p^{e}$, where $p$ is a prime and $e \geq 1$, be such that $q \equiv$ $3(\bmod 4)$. Let $G \cong \mathbb{Z}_{p}^{e} \cdot \mathbb{Z}_{(q-1) / 2}<\mathrm{AGL}\left(1, p^{e}\right)$. Then, the dihedral group $H \cong \mathrm{D}_{q+1}$ is a B-group, $|G|$ and $|H|$ are coprime, and $G \times H$ is not a B-group.

Theorems 1.1 and 1.2 are proved in Subsections 2.3 and 3.2, respectively.

Examples of some other objects will also be constructed, such as vertex-transitive self-complementary tournaments, relative difference sets, and vertex-transitive nonCayley graphs; see Sections 2, 3 and 5.

1.2. Quotients of Cayley graphs. Let $\Gamma$ be a graph with vertex set $V$. For a partition $\mathcal{B}$ of $V$, the quotient graph of $\Gamma$ induced on $\mathcal{B}$ is the graph with vertex set $\mathcal{B}$ such that $B, B^{\prime} \in \mathcal{B}$ are adjacent if and only if some vertex $u \in B$ is adjacent in $\Gamma$ to some vertex $v \in B^{\prime}$. If for all $B \in \mathcal{B}$ and all $v \in B$ the valencies satisfy $\left|\Gamma_{\mathcal{B}}(B)\right|=|\Gamma(v)|$, then $\Gamma$ is called a cover of $\Gamma_{\mathcal{B}}$. Assume that $\Gamma$ is an $X$-vertextransitive graph. If $\mathcal{B}$ is an $X$-invariant partition of $V$ for some transitive subgroup $X$ of Aut $\Gamma$, then the quotient $\Gamma_{\mathcal{B}}$ is called an imprimitive quotient of $\Gamma$; if further $\Gamma$ is a cover of $\Gamma_{\mathcal{B}}$, then $\Gamma$ is said to be an imprimitive cover of $\Gamma_{\mathcal{B}}$. In the case where there exists an intransitive normal subgroup $N \triangleleft X$ such that $\mathcal{B}$ is the set of $N$-orbits in $V$, then $\Gamma_{\mathcal{B}}$ is called a normal quotient of $\Gamma$ and sometimes denoted by $\Gamma_{N}$, and if further $\Gamma$ is a cover of $\Gamma_{N}$, then it is called a normal cover. Let $C=\operatorname{core}_{X}(\hat{G})$, and let $N \leq C$ be a normal subgroup of $X$. Then, the normal quotient $\Gamma_{N}$ is isomorphic to the Cayley graph $\operatorname{Cay}(G / N, S N / N)$. Such a special normal quotient is called a core quotient; if in addition $\Gamma$ is a cover of $\Gamma_{N}$, then $\Gamma$ is called a core cover of $\Gamma_{N}$.

A graph $\Gamma$ is called $X$-arc-transitive or $(X, 2)$-arc-transitive if $X \leq$ Aut $\Gamma$ is transitive on the $\operatorname{arcs}$ or the 2 -arcs of $\Gamma$, respectively. (An arc is an ordered pair of 
adjacent vertices, and a 2-arc is a path of length 2.) An $X$-arc-transitive graph $\Gamma$ is called $X$-locally primitive if $X_{v}$ acts primitively on $\Gamma(v)$, where $v$ is a vertex. By taking a core quotient, we have a characterisation for the class of locally primitive holomorph Cayley graphs. A group $G$ is called quasi-simple if $G=G^{\prime}$, and $G / \mathbf{Z}(G)$ is simple, while a group $G$ is called almost quasi-simple if $T \leq G \leq T$.Out $(T)$ for some quasi-simple group $T$.

Theorem 1.3. Let $\Gamma=\operatorname{Cay}(G, S)$ be connected, and let $X=\hat{G} \circ \tilde{G}$. Assume that $\Gamma$ is $X$-locally primitive of valency at least 3 . Then, $S=s^{G}$ for some involution $s \in G$ such that $\mathbf{C}_{G}(s)$ is a maximal subgroup of $G$, and one of the following holds:

(i) $G=\mathrm{D}_{2 p}$ with $p$ odd prime, and $\Gamma \cong \mathrm{K}_{p, p}$;

(ii) $G$ is quasi-simple;

(iii) $G=T \rtimes\langle s\rangle$ with $T$ quasi-simple, and $\mathbf{C}_{G}(s)=\mathbf{C}_{T}(s) \times\langle s\rangle$;

(iv) $G=(T \circ T) \rtimes\langle s\rangle$ with $T$ quasi-simple, and $\mathbf{C}_{G}(s) \cong T \times\langle s\rangle$. Moreover, if $\Gamma$ is $(X, 2)$-arc transitive, then $G \cong \mathrm{S}_{3}$ and $\Gamma \cong \mathrm{K}_{3,3}$.

See Subsection 4.3 for the proof of Theorem 1.3 This approach also provides a short proof for Praeger's reduction theorem for 2-arc transitive graphs; see Theorem 4.9 and its proof.

However, a normal quotient of a Cayley graph is not necessarily a Cayley graph, and 'most' imprimitive quotients of a Cayley graph are not Cayley graphs.

Proposition 1.4. $\quad$ (i) Each vertex- and edge-transitive graph is a normal quotient of an edge-transitive Cayley graph.

(ii) Each vertex- and edge-transitive graph is an imprimitive quotient of an edge-transitive Cayley graph of valency 2, a disjoint union of cycles of the same size.

See Proposition 5.3 for the proof of this proposition. Our analysis of normal quotients of a Cayley graph also leads to a method for constructing vertex-transitive non-Cayley graphs; see Proposition 5.1 and Example 5.2

1.3. Dual Cayley graphs and rotary Cayley maps. For a Cayley graph $\Gamma=$ Cay $(G, S)$ and a subgroup $X \leq \operatorname{Aut} \Gamma$ with $\hat{G}<X$, if $\hat{G}$ is core-free in $X$, then $X$ has a faithful transitive permutation representation on $[X: \hat{G}]$ with $X_{1}$ being a regular subgroup. Thus, any $X$-vertex transitive graph with vertex set $[X: \hat{G}]$ is a Cayley graph of the group $X_{1}$. Such a graph is called a dual Cayley graph of $\Gamma$. The concept of dual Cayley graphs establishes a connection from rotary Cayley maps to circulants and dihedral Cayley graphs. Thus, permutation groups containing a cyclic or dihedral regular subgroup are important in the study of rotary Cayley maps. A precise list of a primitive permutation group containing a regular cyclic subgroup was recently given by Jones [17] and the author [21] independently, based on the work of Feit [11]. A classical result of Wielandt [36] shows that each primitive permutation group containing a regular dihedral subgroup is 2-transitive. Here we obtain a complete list for those groups, which is justified in Section 7.

Theorem 1.5. Let $X$ be a quasiprimitive permutation group of degree $n$. Then, $X$ contains a dihedral regular subgroup $G$ if and only if $X$ is 2-transitive, and one of the following holds, where $\omega \in \Omega$ :

(i) $(X, G)=\left(\mathrm{A}_{4}, \mathrm{D}_{4}\right),\left(\mathrm{S}_{4}, \mathrm{D}_{4}\right),\left(\operatorname{AGL}(3,2), \mathrm{D}_{8}\right),\left(\mathrm{AGL}(4,2), \mathrm{D}_{16}\right),\left(\mathrm{A}_{6}, \mathrm{D}_{16}\right)$, or $\left(\mathrm{A}_{7}, \mathrm{D}_{16}\right)$; 
(ii) $\left(X, G, X_{\omega}\right)=\left(\mathrm{M}_{12}, \mathrm{D}_{12}, \mathrm{M}_{11}\right),\left(\mathrm{M}_{22} .2, \mathrm{D}_{22}, \mathrm{PSL}(3,4) .2\right)$, or $\left(\mathrm{M}_{24}, \mathrm{D}_{24}, \mathrm{M}_{23}\right)$;

(iii) $\left(X, G, X_{\omega}\right)=\left(\mathrm{S}_{2 m}, \mathrm{D}_{2 m}, \mathrm{~S}_{2 m-1}\right)$ or $\left(\mathrm{A}_{4 m}, \mathrm{D}_{4 m}, \mathrm{~A}_{4 m-1}\right)$;

(iv) $X=\operatorname{PSL}\left(2, p^{e}\right) . O, G=\mathrm{D}_{p^{e}+1}$, and $X_{\omega} \unrhd \mathbb{Z}_{p}^{e} \rtimes \mathbb{Z}_{\frac{p^{e}-1}{2}}$.O, where $p^{e} \equiv 3$ $(\bmod 4)$, and $O \leq \operatorname{Out}\left(\operatorname{PSL}\left(2, p^{e}\right)\right) \cong \mathbb{Z}_{2} \times \mathbb{Z}_{e}$;

(v) $X=\operatorname{PGL}\left(2, p^{e}\right) \cdot \mathbb{Z}_{f}, G=\mathrm{D}_{p^{e}+1}$, and $X_{\omega}=\mathbb{Z}_{p}^{e} \rtimes \mathbb{Z}_{p^{e}-1}$, where $p^{e} \equiv 1$ $(\bmod 4)$, and $f \mid e$.

A map $\mathcal{M}$ is said to be rotary or regular if its automorphism group Aut $\mathcal{M}$ is transitive on arcs or flags, respectively; see [7] for the definitions. A rotary map is said to be chiral if it is not regular. A map $\mathcal{M}$ is called a Cayley map of a group $G$ if Aut $\mathcal{M}$ contains the regular subgroup $\hat{G}$. The study of orientable Cayley maps was initiated by Biggs [1] in 1972, and is a current active topic in topological and algebraic graph theory; see for example $3,8,3,33,34$. Here we apply Theorem 1.5 to give a characterisation of rotary Cayley maps of simple groups.

Theorem 1.6. Every finite simple group has rotary Cayley maps; however, there exist finite simple groups which have no regular Cayley map, and there exist finite simple groups which have no chiral Cayley map. Moreover, for a non-abelian simple group $G$ and a rotary Cayley map $\mathcal{M}$ of $G$, one of the following holds:

(i) $\hat{G} \triangleleft$ Aut $\mathcal{M}$;

(ii) $\mathcal{M}$ is chiral, and the pair (Aut $\mathcal{M}, G$ ) is one of the following:

(PSL $\left.(2,11), \mathrm{A}_{5}\right),\left(\mathrm{M}_{23}, \mathrm{M}_{22}\right)$, or $\left(\mathrm{A}_{2 m+1}, \mathrm{~A}_{2 m}\right)$ with $m$ even;

(iii) $\mathcal{M}$ is regular, and the pair (Aut $\mathcal{M}, G$ ) is one of the following:

\begin{tabular}{|c||c|c|c|c|c|}
\hline $\begin{array}{c}\text { Aut } \mathcal{M} \\
G\end{array}$ & $\mathrm{AGL}(3,2)$ & $2^{4} \rtimes \mathrm{A}_{6}$ & $2^{4} \rtimes \mathrm{A}_{7}$ & $\mathrm{AGL}(4,2)$ & \\
$\mathrm{GL}(3,2)$ & $\mathrm{A}_{6}$ & $\mathrm{~A}_{7}$ & $\mathrm{GL}(4,2)$ & \\
\hline Aut $\mathcal{M}$ & $\mathrm{M}_{12}$ & $\mathrm{M}_{24}$ & $\mathrm{~S}_{2 m+1}$ & $\mathrm{~A}_{2 m+1} \times \mathbb{Z}_{2}$ & $\mathrm{~A}_{4 m}$ \\
$G$ & $\mathrm{M}_{11}$ & $\mathrm{M}_{23}$ & $\mathrm{~A}_{2 m}$ & $\mathrm{~A}_{2 m}$ & $\mathrm{~A}_{4 m-1}$ \\
\hline
\end{tabular}

The first statement in Theorem 1.6 about the existence of rotary Cayley maps of non-abelian simple groups is a straightforward consequence of a result of [27] that each non-abelian simple group is generated by two elements, one of which is an involution. This fact was also observed in 8 independently.

If a Cayley map $\mathcal{M}$ of $G$ is such that $\hat{G} \triangleleft$ Aut $\mathcal{M}$, then $\mathcal{M}$ is called a normal Cayley map. If further $\mathcal{M}$ is orientable, then $\mathcal{M}$ is called balanced. A nice characterization of balanced Cayley maps and their embedding is given in 34. Many known examples of rotary Cayley maps are balanced. The Cayley maps stated in the next proposition show that 'balanced' for Cayley maps is not a topological property.

Proposition 1.7. There exist chiral maps that may be represented as balanced Cayley maps, as well as non-balanced Cayley maps.

This proposition is proved in Subsection 3.3. Taking normal quotient of Cayley graphs also provides a characterisation of rotary Cayley maps of abelian groups; see Proposition 5.5.

\section{Normal CAYley graphs AND their CONStructions}

We study normal Cayley graphs and their construction in this section. We first present some basic properties, and then produce some interesting examples. 
2.1. Basic properties. The first lemma is important for studying normal Cayley graphs, part (1) of which was first proved in [13, Lemma 2.1].

Lemma 2.1. Let $\Gamma=\operatorname{Cay}(G, S)$, and let $H=\left\{h \in G \mid h^{-1} S h=S\right\}$. Then

(1) the normalizer $\mathbf{N}_{\mathrm{Aut} \Gamma}(\hat{G})=\hat{G} \rtimes \operatorname{Aut}(G, S)$;

(2) the centralizer $\mathbf{C}_{\mathrm{Aut} \Gamma}(\hat{G})$ is uniquely determined by $\operatorname{lnn}(G, S)$, and further,

$$
\begin{aligned}
& \hat{G} \mathbf{C}_{\text {Aut } \Gamma}(\hat{G})=\hat{G} \circ \tilde{H}=\hat{G} \rtimes \operatorname{Inn}(G, S), \quad \text { and } \\
& \operatorname{lnn}(G, S)=\{\hat{h} \tilde{h} \mid h \in H\} \cong \tilde{H} /(\hat{H} \cap \tilde{H}) .
\end{aligned}
$$

Proof. We only need to prove part (2). The equality $\hat{G} \mathbf{C}_{\text {Aut } \Gamma}(\hat{G})=\hat{G} \rtimes \operatorname{lnn}(G, S)$ was proved in [20, Lemma 3.6]. It is known that $N:=\hat{G} \mathbf{C}_{\operatorname{Sym}(G)}(\hat{G})=\hat{G} \circ \tilde{G}$; see 44. Since $\hat{G}$ is regular on $G$ and normal in $N$, we have that $N=\hat{G} \rtimes N_{\mathbf{1}}$. Further, $N_{\mathbf{1}}=\{\hat{g} \tilde{g} \mid g \in G\}=\operatorname{lnn}(G)$, and an element $\hat{g} \tilde{g}$ lies in Aut $\Gamma$ if and only if it fixes $S$. Since $H=\left\{h \in G \mid h^{-1} S h=S\right\}$, we have $N_{\mathbf{1}} \cap \operatorname{Aut} \Gamma=\{\hat{h} \tilde{h} \mid h \in H\}=\operatorname{Inn}(G, S)$. So

$$
\hat{G} \mathbf{C}_{\text {Aut } \Gamma}(\hat{G})=N \cap \operatorname{Aut} \Gamma=\hat{G} \cdot\left(N_{\mathbf{1}} \cap \operatorname{Aut} \Gamma\right)=\langle\hat{g}, \hat{h} \tilde{h} \mid g \in G, h \in H\rangle=\hat{G} \circ \tilde{H},
$$
and $\operatorname{lnn}(G, S) \cong N / \hat{G} \cong \hat{G} \tilde{H} / \hat{G} \cong \tilde{H} /(\hat{G} \cap \tilde{H})$.

Hence, the subgroups $\mathbf{N}_{\mathrm{Aut} \Gamma}(\hat{G})$ and $\hat{G} \mathbf{C}_{\mathrm{Aut} \Gamma}(\hat{G})$ of the automorphism group Aut $\Gamma$ can be described in terms of $\operatorname{Aut}(G, S)$ and $\operatorname{lnn}(G, S)$, respectively. The subgroup $\operatorname{Aut}(G, S)$ has thus played a significant role in the study of Cayley graphs in the literature; see for example [13, 32, 37.

Assume that $\Gamma=\operatorname{Cay}(G, S)$ is such that $\hat{G} \triangleleft X \leq \operatorname{Aut} \Gamma$. Then, by Lemma 2.1 the vertex stabiliser $X_{1} \leq \operatorname{Aut}(G, S)$. Thus, $X_{1}$ acts on $\Gamma$ in a particular nice way - by conjugation. In particular, if $\Gamma$ is connected, then the action of $X_{1}$ on $\Gamma$ is uniquely determined by its action on $\Gamma(\mathbf{1})=S$. Due to this nice action of $X$, various properties of an $X$-normal Cayley graph can be identified easily, as indicated in the next lemma [19, 32].

Lemma 2.2. Let $G$ be a group, and let $\Gamma=\operatorname{Cay}(G, S)$ be a connected $(X, r)$-arc transitive graph, where $r=1 / 2$ or $r$ is a positive integer. Assume that $\hat{G}$ is normal in $X$. Then, $r=1 / 2,1$ or $2, X_{1}$ is a permutation group of $S$, and the following statements hold:

(i) for any $g, h \in S$ with $g \neq h^{-1}$, there exists $\sigma \in \operatorname{Aut}(G, S)$ such that $g^{\sigma}=h$ or $h^{-1}$

(ii) for $r=1$, all elements of $S$ are conjugate under $\operatorname{Aut}(G, S)$;

(iii) if $X_{1}$ acts primitively on $S$, all elements of $S$ are involutions;

(iv) for $r=2, X_{1}$ is a 2-transitive permutation group on $S$; in particular, Aut $(G, S)$ is a 2-transitive permutation group on $S$.

Lemma 2.1 suggests a method for constructing Cayley graphs with 'many' automorphisms:

Take a subgroup $H \leq \operatorname{Aut}(G)$ and choose elements $s_{1}, s_{2}, \ldots, s_{m} \in$ $G$. Let $S=\left\{h^{-1} s_{i} h \mid 1 \leq i \leq m, h \in H\right\}$, and set $\Gamma=\operatorname{Cay}(G, S)$.

Observe that Aut $(G, S)$ then contains the inner automorphisms of $G$ induced by elements of $H$. In particular, if $m=1$, then $\Gamma$ is arc-transitive; while if $m=2$ and $s_{2}=s_{1}^{-1}$, then $\Gamma$ is undirected and edge-transitive. Such a Cayley graph is a normal edge-transitive Cayley graph; see [32]. 
The following example shows that the structure of the subgroups $\operatorname{Aut}(G, S)$ and $\operatorname{lnn}(G, S)$ depends not only on the graph $\Gamma$ but also on the base group $G$. Thus, the property for a Cayley graph $\operatorname{Cay}(G, S)$ to be normal edge-transitive is not a 'graph theoretic' property since it also depends on the choice of the base group $G$.

Example 2.3. Let $\Gamma=\mathbf{Q}_{d}$ be the hypercube of dimension $d$. Then, Aut $\Gamma \cong \mathbb{Z}_{2}^{d} \cdot \mathrm{S}_{d}$. For any group with form $G:=G_{1} \times G_{2} \times \cdots \times G_{l}$ such that $|G|=2^{d}$ and each $G_{i} \cong \mathbb{Z}_{2}, \mathbb{Z}_{4}$ or $\mathrm{D}_{8}$, it is easily shown that the graph $\Gamma$ may be represented as a Cayley graph of the group $G$, that is, $\Gamma \cong \operatorname{Cay}(G, S)$ for some $S \subset G$. If we choose $G$ to be the normal elementary abelian 2-group $\mathbb{Z}_{2}^{d}$ of $\operatorname{Aut} \Gamma$, then $\operatorname{Aut}(G, S) \cong \mathrm{S}_{d}$; if $G=\mathbb{Z}_{4}^{d / 2}$ with $d$ even, then $\operatorname{Aut}(G, S)$ may be transitive but must be imprimitive on $S$; while in other cases, Aut $(G, S)$ is not transitive on $S$.

2.2. The holomorph Cayley digraphs which are self-complementary tournaments. Let $X$ be a transitive permutation group on a set $\Omega$. The digraph with vertex set $\Omega$ and with edge set $\left\{(\alpha, \beta)^{x} \mid x \in X\right\}$ where $\alpha, \beta \in \Omega$ is called an orbital of $X$. The orbital $\left\{(\beta, \alpha)^{x} \mid x \in X\right\}$ is called the paired orbital of the orbital $\left\{(\alpha, \beta)^{x} \mid x \in X\right\}$.

Let $G$ be a group, and let $s_{1}, s_{2}, \ldots, s_{m} \in G \backslash\{1\}$. Let $S=\left\{s_{i}^{g} \mid 1 \leq i \leq m, g \in\right.$ $G\}$, the union of the full conjugacy class of the elements $s_{i}$. Let $\Gamma=\operatorname{Cay}(G, S)$. Then, $\Gamma$ is a holomorph Cayley digraph, and $X:=\hat{G} \rtimes \operatorname{lnn}(G) \leq \operatorname{Aut} \Gamma$. For holomorph Cayley digraphs, there is a special permutation $\tau$ of vertices associated with $X$, given by

$$
\tau: \quad x \rightarrow x^{-1}, \text { for } x \in G .
$$

Lemma 2.4. The permutation $\tau$ maps each orbital of $X$ to its paired orbital.

Proof. Let $\Sigma$ be an orbital of $X$ and $\Sigma^{*}$ the paired orbital of $\Sigma$. Then, there exists a subset $S \subset G$ such that $\Sigma=\operatorname{Cay}(G, S)$ and $\Sigma^{*}=\operatorname{Cay}\left(G, S^{-1}\right)$. By the definition, for any elements $a, b \in G,(a, b)$ is an arc of $\Sigma$ if and only if $b a^{-1} \in S$. We claim that the ordered pair $\left(a^{\tau}, b^{\tau}\right)$ is an arc of $\Sigma^{*}$. Indeed, we have $\left(a^{\tau}, b^{\tau}\right)=\left(a^{-1}, b^{-1}\right)$, and $b^{-1}\left(a^{-1}\right)^{-1}=b^{-1} a=\left(a^{-1} b\right)^{-1}=\left(\left(b a^{-1}\right)^{a}\right)^{-1} \in S^{-1}$. Therefore, $\tau$ maps arcs of $\Sigma$ to arcs of $\Sigma^{*}$, and $\tau$ is an isomorphism from $\Sigma$ to $\Sigma^{*}$.

The next corollary collects some properties regarding holomorph Cayley graphs.

Corollary 2.5. Let $\Gamma=\operatorname{Cay}(G, S)$ be a holomorph graph, and let $X=\hat{G} \rtimes \operatorname{lnn}(G)$. Then the following hold:

(i) $\Gamma$ is undirected if and only if $S=\left\{s, s^{-1}\right\}^{G}$ for some element $s$;

(ii) $\Gamma$ is $X$-arc-transitive;

(iii) the stabiliser $X_{\mathbf{1} s} \cong \mathbf{C}_{G}(s) / \mathbf{Z}(G)$, and the action of $X_{\mathbf{1}}$ on $S$ is equivalent to the action of $G$ on $\left[G: \mathbf{C}_{G}(s)\right]$;

(iv) $\Gamma$ is $X$-locally primitive if and only if $S=s^{G}$ for an involution $s$, and $\mathbf{C}_{G}(s)$ is a maximal subgroup of $G$.

Proof. (i) It follows from the definition.

(ii) If $\Gamma$ is not undirected, then by definition, it is $X$-arc transitive; on the other hand, if $\Gamma$ is undirected, then by definition, $\Gamma$ is $X$-edge transitive, and further, by Lemma 2.4. $\Gamma$ is $X$-arc transitive.

(iii) The stabiliser $X_{\mathbf{1}}$ is equal to $\left\langle\sigma \in \operatorname{lnn}(G) \mid s^{\sigma}=s\right\rangle$, that is, $X_{\mathbf{1} s}$ consists of all inner automorphisms of $G$ induced by elements of $G$ which centralise $s$. It follows that $X_{\mathbf{1} s} \cong \mathbf{C}_{G}(s) / \mathbf{Z}(G)$. 
(iv) By Lemma 2.2(iii), there exists an involution $s$ such that $S=s^{G}$. Since $X_{1}$ is primitive on $S$, the stabiliser $X_{1 s}$ is a maximal subgroup of $X_{\mathbf{1}}$. It then follows that $\mathbf{C}_{G}(s)$ is a maximal subgroup of $G$.

The following construction can produce some very interesting graphs.

Construction 2.6. Let $G$ be a group that has exactly $2 l$ conjugacy classes of elements of order at least 3 :

$$
s_{1}^{G},\left(s_{1}^{-1}\right)^{G}, \ldots, s_{l}^{G},\left(s_{l}^{-1}\right)^{G} .
$$

Let $S$ be a subset of $G$ which contains exactly one of $s_{i}^{G}$ and $\left(s_{i}^{-1}\right)^{G}$ as a subset for each $i=1,2, \ldots, l$. Let $\Gamma=\operatorname{Cay}(G, S)$.

A tournament is a digraph $\Gamma$ such that for any two vertices $v, w$, exactly one of the ordered pairs $(v, w)$ and $(w, v)$ is an arc of $\Gamma$. The complement $\bar{\Gamma}$ of a digraph $\Gamma$ is the digraph with vertex set $V \Gamma$, and $(v, w)$ is an arc of $\bar{\Gamma}$ if and only if it is not an arc of $\Gamma$. A digraph is called self-complementary if it is isomorphic to its complement. The next proposition characterises the holomorph Cayley digraphs which are self-complementary tournaments.

Proposition 2.7. Let $\Gamma=\operatorname{Cay}(G, S)$ be a holomorph Cayley digraph. Then, $\Gamma$ is a self-complementary tournament if and only if $G$ has odd order and $\Gamma$ is as in Construction 2.6.

Consequently, a group of odd order with $2 l$ conjugacy classes of non-identity elements has exactly $2^{l}$ different holomorph Cayley digraphs which are self-complementary tournaments.

Proof. Assume that $\Gamma$ is a Cayley digraph as in Construction 2.6. Then $G \backslash(S \cup$ $\{1\})=S^{-1}$ and $S^{\tau}=S^{-1}$. Thus, $\bar{\Gamma}=\operatorname{Cay}\left(G, S^{-1}\right)$ is the complement of $\Gamma$, and $\tau$ induces an isomorphism between $\Gamma$ and $\bar{\Gamma}$. So $\Gamma$ is self-complementary. For any two elements $x, y \in G$, exactly one of the elements $y x^{-1}$ and $x y^{-1}$ belongs to $S$. Thus, exactly one of the pairs $(x, y)$ and $(y, x)$ is an arc of $\Gamma$, so $\Gamma$ is a tournament.

Conversely, assume that a holomorph Cayley digraph $\Gamma$ is a self-complementary tournament. Then, for any two elements $x, y \in G$, exactly one of the ordered pairs $(x, y)$ and $(y, x)$ is an arc of $\Gamma$ since $\Gamma$ is a tournament. Thus, for any nonidentity element $g \in G$, exactly one of $g$ and $g^{-1}$ belongs to $S$. In particular, $g$ is not an involution, and so $G$ has odd order. Since $\Gamma$ is a holomorph, exactly one of the conjugacy classes $g^{G}$ and $\left(g^{-1}\right)^{G}$ is a subset of $S$. Thus, $\Gamma$ is as in Construction 2.6 .

Let $\mathrm{K}_{r ; 2}$ be a complete $r$-partite graph with parts of size 2. An almost tournament of order $2 r$ is a sub-digraph $\Gamma$ of $\mathrm{K}_{r ; 2}$ such that for any edge $\{v, w\}$ of $\mathrm{K}_{r ; 2}$, exactly one of the ordered pairs $(v, w)$ and $(w, v)$ is an arc of $\Gamma$. A digraph $\Gamma$ of order $2 r$ is called almost self-complementary if $\Gamma<\mathrm{K}_{r ; 2}$ and $\Gamma \cong \mathrm{K}_{r ; 2}-\Gamma$. The next proposition characterises the holomorph Cayley digraphs which are almost self-complementary tournaments, the proof of which is similar to the proof of Proposition 2.7.

Proposition 2.8. Let $\Gamma=\operatorname{Cay}(G, S)$ be a holomorph Cayley digraph. Then, $\Gamma$ is an almost self-complementary tournament if and only if $G$ has only one subgroup of order 2 and $\Gamma$ is as in Construction 2.6. 
Proof. Assume that $G$ is a group of order $2 r$ with $r$ odd, and that $\Gamma$ is a Cayley digraph as in Construction [2.6. Let $I$ be the unique subgroup of $G$ of order 2 . Then, $G \backslash(S \cup I)=S^{-1}$ and $S^{\tau}=S^{-1}$. Thus, $\mathrm{K}_{r ; 2}-\Gamma=\operatorname{Cay}\left(G, S^{-1}\right)$, and $\tau$ induces an isomorphism between $\Gamma$ and $\mathrm{K}_{r ; 2}-\Gamma$. It follows that $\Gamma$ is almost self-complementary. For any edge $\{x, y\}$ of $\mathrm{K}_{r ; 2}$, exactly one of the elements $y x^{-1}$ and $x y^{-1}$ belongs to $S$. Therefore, exactly one of the pairs $(x, y)$ and $(y, x)$ is an arc of $\Gamma$, hence $\Gamma$ is an almost tournament.

Conversely, assume that a holomorph Cayley digraph $\Gamma$ is an almost self-complementary tournament. Then, for any edge $\{x, y\}$ of $\mathrm{K}_{r ; 2}$, exactly one of the ordered pairs $(x, y)$ and $(y, x)$ is an arc of $\Gamma$ since $\Gamma$ is an almost tournament. Thus, for any element $g \in G$ of order at least 3, exactly one of $g$ and $g^{-1}$ belongs to $S$. In particular, $G$ has only one subgroup of order 2 . Since $\Gamma$ is a holomorph, exactly one of the conjugacy classes $g^{G}$ and $\left(g^{-1}\right)^{G}$ is a subset of $S$. Thus, $\Gamma$ is as in Construction 2.6.

2.3. A class of half-transitive normal Cayley graphs. Here we consider vertex-primitive edge-transitive graphs of twisted wreath product type; see [4 for the detailed description of primitive permutation groups of twisted wreath product type.

Let $X$ be an arbitrary group, and assume that $G$ is an insoluble minimal normal subgroup of $X$, say $G=T_{1} \times \cdots \times T_{l}=T^{l}$, where $l \geq 1$ and $T_{i} \cong T$ is non-abelian simple. For an element $g=t_{1} t_{2} \ldots t_{l} \in G$ such that $t_{i} \in T_{i}$, let

$$
M(g)=\prod_{\left\{i \mid t_{i} \neq 1\right\}} T_{i}, \quad l(g)=\left|\left\{i \mid t_{i} \neq 1\right\}\right| .
$$

Then, $M(g)$ is the smallest normal subgroup of $G$ which contains the element $g$, and $l(g)$ is the number of direct factors of $M(g)$.

Lemma 2.9. For each element $g \in G$ and for each element $x \in X, M\left(g^{x}\right)=M(g)$ if and only if $x$ normalizes $M(g)$; in particular, if $g^{x}=g^{-1}$, then $x$ normalizes $M(g)$.

Proof. For an element $g \in G$ and an element $x \in X$, if $x$ normalizes $M(g)$, then clearly $M\left(g^{x}\right)=M(g)$.

Conversely, assume that, for some element $g \in G$ and some element $x \in X$, $M\left(g^{x}\right)=M(g)$. Then $g^{x} \in M(g)$, and thus $g \in M(g)^{x^{-1}}$. Since $M(g) \triangleleft G$, we have $M(g)^{x^{-1}} \triangleleft G^{x^{-1}}=G$, and so $g \in M(g)^{x^{-1}} \cap M(g) \triangleleft G$. Since $M(g)$ is the smallest normal subgroup of $G$ containing $g$, we have that $M(g)^{x^{-1}} \cap M(g)=M(g)$, and hence $x$ normalizes $M(g)$. Finally, if $g^{x}=g^{-1}$, then $x$ normalizes $M\left(g^{-1}\right)=$ $M(g)$.

We are ready to give a characterization for a vertex-primitive edge-transitive graph of twisted wreath product type to be arc-transitive.

Theorem 2.10. Let $X$ be a primitive permutation group on a set $\Omega$ of twisted wreath product type, and let $\Gamma=\Sigma \cup \Sigma^{*}$, where $\Sigma$ is an orbital of $X$ and $\Sigma^{*}$ is the paired orbital of $\Sigma$. Identify $\Gamma$ with the graph $\operatorname{Cay}(G, S)$, where $G=\operatorname{soc}(X)$ and $S \subset G$. Then, $\Gamma$ is $X$-arc transitive if and only if each element $g \in S$ is conjugate to $g^{-1}$ in $\mathbf{N}_{X_{1}}(M(g))$. In particular, if some element $g \in S$ is not conjugate in Aut $(M(g))$ to $g^{-1}$, then $\Gamma$ is half-transitive. 
Proof. Now $G=T_{1} \times \cdots \times T_{l}$ is the unique minimal normal subgroup of $X$, where $l \geq 5$ and $T_{1} \cong \ldots \cong T_{l} \cong T$ is a non-abelian simple group, and $X=G \rtimes X_{1}$. It follows from Lemma 2.9 that $\Gamma$ is $X$-arc transitive if and only if for each element $g \in S, g$ is in $\mathbf{N}_{X_{1}}(M(g))$ conjugate to $g^{-1}$.

Let $Y=$ Aut $\Gamma$. Then $X \leq Y$, and $Y$ is primitive on $G$. By [30, $Y$ is a primitive permutation group of twisted wreath product type with socle $G$, and thus $Y=G \rtimes Y_{\mathbf{1}}$. Suppose that $\Gamma$ is arc transitive. Then, $Y_{\mathbf{1}}$ acts transitively on $S$; in particular, $g^{-1}=g^{\sigma}$ for some $\sigma \in Y_{1} \leq \operatorname{Aut}(G, S)$. By Lemma 2.9, $\sigma$ normalizes $M(g)$, that is, $\sigma \in \mathbf{N}_{Y_{1}}(M(g))$. Hence, $\sigma$ induces an automorphism of $M(g)$ which fuses $g$ and $g^{-1}$. Therefore, if $g$ is not fused in Aut $(M(g))$ to $g^{-1}$, then $\Gamma$ is half-transitive.

We observe that Theorem 1.1 is a special case with $g$ being an element of $T$. Thus, the proof of Theorem 1.1 follows.

With Theorem 1.1, it is easy to construct half-transitive graphs of large valency.

Example 2.11. Let $T=\operatorname{Sz}(q)$ with $q=2^{2 m+1} \geq 8$, and let $t$ be an element of $T$ of order 4. Then, $t$ is not conjugate in $\operatorname{Aut}(T)$ to $t^{-1}$. Let $X$ be a primitive permutation group on $\Omega$ of twisted wreath product type such that $G:=\operatorname{soc}(X)=$ $T^{k}$. Identify $\Omega$ with $G$, so that $X=G \rtimes X_{1}$ and $X_{1}$ acts on $G$ by conjugation. Let $S=\left\{h^{-1} t h, h^{-1} t^{-1} h \mid h \in X_{1}\right\}$. Then, the graph $\Gamma=\operatorname{Cay}(G, S)$ is connected and $X$-edge-transitive. Further, by Theorem 1.1, $\Gamma$ is half-transitive.

\section{CAYley gRAPHS AS COSET GRAPHS}

In this section, we investigate a method for constructing core-free Cayley graphs.

3.1. Coset graphs. Let $X$ be a group. For a core-free subgroup $H$ of $X$ and a subset $S \subset X$, let $[X: H]=\{H x \mid x \in X\}$, and define the coset graph $\Gamma:=$ $\operatorname{Cos}(X, H, H S H)$ as the graph with vertex set $[X: H]$ such that $(H x, H y)$ is an arc of $\Gamma$ if and only if $y x^{-1} \in H S H$. This following lemma collects basic properties for a typical method in the constructions of vertex-transitive graphs; see for example 16.

Lemma 3.1. Let $\Gamma=\operatorname{Cos}(X, H, H S H)$. Then the following statements hold:

(i) $\Gamma$ is $X$-vertex-transitive;

(ii) $\Gamma$ is connected if and only if $\langle H, S\rangle=X$;

(iii) $\Gamma$ is undirected if and only if $H S H=H S^{-1} H$;

(iv) $\Gamma$ is $X$-edge-transitive if and only if $H S H=H\left\{g, g^{-1}\right\} H$, where $g \in X$;

(v) $\Gamma$ is undirected and $X$-arc-transitive if and only if $H S H=H g H$ with $g^{2} \in H$

(vi) $\Gamma$ is a Cayley graph of a group $G$ such that $\hat{G} \leq X$ if and only if $X=\hat{G} H$ and $\hat{G} \cap H=1$.

This provides us with a method for constructing edge-transitive Cayley graphs of a given group $G$.

Construction 3.2. For a group $G$, find a larger group $X$ such that $X=G H, G \cap$ $H=1$ and $H$ is core-free in $X$. Take $g \in X$, and let $\Gamma=\operatorname{Cos}\left(X, H, H\left\{g, g^{-1}\right\} H\right)$.

For a Cayley graph $\Gamma$ of a group $G$ constructed as a coset graph, we know that there exists some subset $S \subset G$ such that $\Gamma=\operatorname{Cay}(G, S)$, but sometimes it is hard 
to list elements of $S$ explicitly in the language of $G$. Here are some examples where the subset $S$ is 'hidden'.

Example 3.3. Let $X=A_{5}$, acting on $\Omega=\{1,2,3,4,5\}$. Let $H=\langle h\rangle$ with $h=$ (12345), and let $s=(12)(34)$. Set $\Sigma=\operatorname{Cos}(X, H, H s H)$. Then, $\Sigma$ is isomorphic to the icosahedron.

On the other hand, let $G=\left\{x \in X \mid 5^{x}=5\right\}$, the stabilizer of 5 in $X$, and let

$$
S=\{(12)(34),(234),(243),(123),(132)\} .
$$

Then, $G=\mathrm{A}_{4}, S \subset G$, and $\Sigma \cong \operatorname{Cay}(G, S)$.

This example tells us that the icosahedron may be explicitly constructed as a coset graph of $A_{5}$, as well as a Cayley graph of $A_{4}$. As a coset graph of $A_{5}$, it is clear that the icosahedron is edge-transitive; however, if we are only given the base group $\mathrm{A}_{4}$ and the subset $S$, it is not easy to see the edge-transitivity.

Example 3.4. Let $G \cong \mathrm{A}_{5}$. The subgroup $\hat{G}$ of $\operatorname{Sym}(G)$ has an overgroup $X \cong$ $\operatorname{PSL}(2,29)$ such that $X=\hat{G} H$ and $\hat{G} \cap H=1$, where $H \cong \mathbb{Z}_{29} \cdot \mathbb{Z}_{7}$. There exists an involution $g \in X$ such that the coset graph $\Gamma=\operatorname{Cos}(X, H, H g H)$ is an arc transitive graph of valency 29. Further, $\Gamma$ is a Cayley graph of $G=\mathrm{A}_{5}$. This construction leads to a proof that the so-called CI-groups are soluble; see [18, 20]. There exists some subset $S \subset G$ such that $\Gamma=\operatorname{Cay}(G, S)$. The subset $S$ is further proved in [5] to be a relative difference set of $A_{5}$. This is the first example of a reversible relative difference set found in an insoluble group. However, the subset $S$ is hidden, and with the assistance of a computer, $S$ is listed below:

(13542), (154), (14)(23), (13254), (12543), (15324), (245), (132),

(152), (13)(45), (12435), (235), (15234), (15342), (125), (14)(25),

(13)(25), (123), (14325), (23)(45), (14235), (253), (254), (13452),

(12453), (145), (12)(35), (14523), (15)(24).

But even with the list of elements of $S$, the structural information of the graph is still hard to read from $G$ and $S$. For instance, it is hard to see whether the graph $\operatorname{Cay}(G, S)$ is arc transitive and whether $S$ is a relative difference set of $\mathrm{A}_{5}$. This is because such properties are 'possessed' by the automorphism group Aut $\Gamma$ but not by the base group $G$ and its automorphism group Aut $(G)$.

This example suggests that some types of Cayley graphs should perhaps more naturally be defined as coset graphs of some groups of order properly divisible by the order of the graphs; see [6, 22, 38, for such examples of constructing $s$-arc transitive Cayley graphs with $s \geq 4$.

3.2. Proof of Theorem 1.2. A permutation group may contain different regular subgroups, and thus a graph may be represented as a Cayley graph of different groups.

Lemma 3.5. Assume that $G$ is a group with $G=H L$ such that $H \cap L=1$. Then, $X:=\operatorname{Hol}(G)=\hat{G} \circ \tilde{G}$ is a transitive permutation group on the set $G$ such that $X_{1}=\operatorname{lnn}(G) \cong G / \mathbf{Z}(G)$, and $X$ has at least four regular subgroups: $\hat{G}, \tilde{G}, \hat{H} \times \tilde{L}$, and $\tilde{H} \times \hat{L}$.

Proof. Recall that $\hat{G}, \tilde{G}$ are subgroups of $\operatorname{Sym}(G)$ acting regularly on the set $G$, and moreover, $X:=\langle\hat{G}, \tilde{G}\rangle=\hat{G} \circ \tilde{G}$. It is easily shown that $X_{1}=\operatorname{lnn}(G)$. 
Assume that $G=H L$ such that $H \cap L=1$. Let $Y=\langle\tilde{H}, \hat{L}\rangle$. Then each element of $\tilde{H}$ commutes with all elements of $\hat{L}$, and hence $Y=\tilde{H} \circ \hat{L}$. Since $H \cap L=1$, it follows that $\tilde{H} \cap \hat{L}=1$. Thus, $Y=\tilde{H} \times \hat{L}$; in particular, $|Y|=|G|$. Let $g$ be an element of $G$. Since $G=H L$, we have $g=h l$ for some element $h \in H$ and some element $l \in L$. Now the element $\left(\tilde{h}^{-1}, \hat{l}\right) \in Y$ maps the vertex 1 to the vertex $g$. Thus, $Y$ is transitive and so is regular on $G$. Similarly, we obtain that $\hat{H} \times \tilde{L}$ is also a regular group on $G$.

We note that, in Lemma 3.5. $\hat{H} \times \tilde{L}$ and $\tilde{H} \times \hat{L}$ are not normal in $X$, but subgroups $\hat{G}$ and $\tilde{G}$ are normal in $X$. Thus, a generalized orbital graph of $X$ may be represented as a normal Cayley graph of $G$ and a Cayley graph of $H \times L$ which is not normal.

With Lemma 3.5, the truth of Theorem 1.2 is easily justified.

Proof of Theorem 1.2. Let $q=p^{e}$, where $p$ is a prime and $e \geq 1$, be such that $q \equiv 3$ $(\bmod 4)$. Let $T=\operatorname{PSL}(2, q)$. Then $T$ has subgroups $G, H$, where $G \cong \mathbb{Z}_{p}^{e} \rtimes \mathbb{Z}_{\frac{q-1}{2}}$ and $H \cong \mathrm{D}_{q+1}$, such that $T=G H$ and $(|G|,|H|)=1$.

By a result of Wielandt (see [36, Theorem 25.6(i)]), the dihedral group $H$ is a B-group.

Let $Y=\hat{T} \times \tilde{T}<\operatorname{Sym}(T)$, and let $L=\hat{G} \times \tilde{H}$. Then, $Y$ is a permutation group on the set $T$ which is primitive but not 2-transitive. By Lemma 3.5, the group $L$ is a regular subgroup of $Y$. Therefore, $L$ is not a B-group.

3.3. A family of Cayley maps that are balanced and non-balanced. The next proposition shows that the property for a Cayley map to be balanced is not a topological property of the map.

Proposition 3.6. Let $p$ be an odd prime $p$ and e a positive integer. Let $\Gamma=\mathrm{K}_{p}\left[\overline{\mathrm{K}}_{p^{e}}\right]$ be a complete p-partite graph with all parts of size $p^{e}$. Then, $\Gamma$ may be embedded into an orientable surface as a rotary Cayley map $\mathcal{M}$, and further, the following statements hold:

(i) $\mathcal{M}$ is chiral, and Aut $\mathcal{M}=\mathbb{Z}_{p^{e+1}} \rtimes \mathbb{Z}_{p^{e}(p-1)}$;

(ii) there exist two cyclic groups $G$ and $H$ of order $p^{e+1}$ such that $\mathcal{M}$ may be represented as a balanced Cayley map of $G$ and a non-balanced Cayley map of $H$;

(iii) a cyclic group $G \cong \mathbb{Z}_{p^{e+1}}$ has two isomorphic chiral Cayley maps which are isomorphic to $\mathcal{M}$ such that one of them is balanced and the other is not balanced.

Proof. Let $G=\langle g\rangle \cong \mathbb{Z}_{p^{e+1}}$, and let $S=\left\{x \in G \mid o(x)=p^{e+1}\right\}$. Then, the Cayley graph Cay $(G, S) \cong \Gamma=\mathrm{K}_{p}\left[\overline{\mathrm{K}}_{p^{e}}\right]$. Let $\rho \in \operatorname{Aut}(G)$ be of order $p^{e}(p-1)$. Then, $\rho \in \operatorname{Aut}(G, S)$, and $\langle\rho\rangle$ acts regularly on $S$. Thus, the group $X:=\langle\hat{G}, \rho\rangle=\hat{G} \rtimes\langle\rho\rangle$, and the graph $\Gamma$ is $X$-arc transitive. By [34, with the rotation $\rho$, the Cayley graph $\Gamma$ has a rotary embedding into an orientable surface as a balanced Cayley map $\mathcal{M}$ of the cyclic group $G$ such that $X \leq$ Aut $\mathcal{M}$.

Since $X$ acts transitively on arcs of the map $\mathcal{M}$, the subgroup $X$ has index at most 2 in Aut $\mathcal{M}$. It is then easily shown that $\left\langle\hat{g}^{p}\right\rangle$ is a normal subgroup of Aut $\mathcal{M}$ and fixes each of the $p$-parts of the complete $p$-partite graph $\Gamma=\mathrm{K}_{p}\left[\overline{\mathrm{K}}_{p^{e}}\right]$. Now the normal quotient of $\Gamma$ induced by $\left\langle\hat{g}^{p}\right\rangle$ is isomorphic to the complete graph $\mathrm{K}_{p}$. If $\mathcal{M}$ is a regular map, then the corresponding quotient map of $\mathcal{M}$ is also regular, 
which is a contradiction; see [1]. Hence, $\mathcal{M}$ is chiral, and in particular Aut $\mathcal{M}=X$, as in part (i).

Furthermore, it is easily shown that the subgroup

$$
F:=\left\langle\hat{g} \rho^{p-1}\right\rangle<X=\text { Aut } \mathcal{M}
$$

is cyclic and acts regularly on the vertex set of $\mathcal{M}$. Thus, $\mathcal{M}$ may be represented as a Cayley map of a cyclic group $H$ corresponding $F=\left\langle\hat{g} \rho^{p-1}\right\rangle$, such that $F=\hat{H}$. Since $F=\hat{H}$ is not normal in Aut $\mathcal{M}, \mathcal{M}$ is not balanced with respect to this base group $H$, as in part (ii).

There exists a subset $R$ of the group $H$ such that $\Gamma=\operatorname{Cay}(H, R)$. Let $\tau$ be an isomorphism from $H$ to $G$, and let $S^{\prime}=R^{\tau}$. Then, $\Gamma \cong$ Cay $\left(G, S^{\prime}\right)$, which has a rotary embedding $\mathcal{M}^{\prime}$. Therefore, $G$ has two isomorphic chiral Cayley maps $\mathcal{M}$ and $\mathcal{M}^{\prime}$ such that $\mathcal{M}$ is balanced and $\mathcal{M}^{\prime}$ is non-balanced with respect to $G$, as in part (iii).

Remark. In Proposition 3.6, if the map $\mathcal{M}$ is represented as a Cayley map of the base group $G$, then it is balanced, and so a nice description of the embedding exists in terms of the base group $G$; see 34 . However, if $\mathcal{M}$ is represented as a Cayley map of the base group $H$, then the embedding does not admit a 'nice' description. Therefore, the choice of base groups of Cayley maps is important in the study of Cayley maps.

3.4. Dual Cayley graphs and rotary Cayley maps. Let $\Gamma=\operatorname{Cay}(G, S)$, and assume that $\hat{G}<X \leq$ Aut $\Gamma$. Assume further that $\hat{G}$ is core-free in $X$, that is, $\Gamma$ is a core-free Cayley graph of $G$. The most important property of core-free Cayley graphs is the following:

$$
X=\hat{G} H, \hat{G} \cap H=1 \text {, and } \hat{G}, H \text { are both core-free, }
$$

where $H=X_{1}$. This implies that $X$ has two faithful transitive permutation representations, that is, on $[X: \hat{G}]$ and $[X: H]$. Further, $X_{1}$ is a regular subgroup of $X$ acting on $[X: \hat{G}]$. In particular, the order $|X|$ is bounded from above in terms of $\left|X_{1}\right|$. This property was used in $[22$ to show that there are only finitely many 2-arc-transitive core-free Cayley graphs of given valency, and there are only finitely many vertex-primitive Cayley graphs of given valency which are not normal. Here the two different actions of $X$ allow us to establish a connection between Cayley maps and circulants and dihedral Cayley graphs. (A Cayley digraph of a cyclic group is called a circulant.)

Lemma 3.7. Let $\mathcal{M}$ be a rotary Cayley map of a group $G$. Assume that $\hat{G}$ is corefree in Aut $\mathcal{M}$. Then, Aut $\mathcal{M}$ acts faithfully on $[$ Aut $\mathcal{M}: \hat{G}]$ and contains a cyclic or dihedral regular subgroup.

Proof. Let $\Gamma$ be a Cayley graph of a cyclic group $G$. Assume that $\hat{G}<X \leq$ Aut $\Gamma$ is such that $\hat{G}$ is core-free in $X$. Then $X=\hat{G} H$ such that $\hat{G} \cap H=1$ where $H=X_{1}$, and $X$ acts faithfully on $[X: \hat{G}]$ such that $H$ is regular on $[X: \hat{G}]$. Taking $X=$ Aut $\mathcal{M}$, we have that $H=X_{1}$ is cyclic or dihedral, as claimed.

This lemma enables us to construct some regular and chiral Cayley maps which are neither balanced nor anti-balanced; see [34, 35, for the definition. Such Cayley maps have been sought for a long time; see 8, 33]. We first construct an infinite family of regular Cayley maps. 
Example 3.8. Let $q=p^{e}$, where $p$ is a prime and $e \geq 1$, be such that $q \equiv$ $3(\bmod 4)$. Let $X=\operatorname{PSL}(2, q)$. Then, $T$ has two subgroups $G$ and $H, G \cong$ $\mathbb{Z}_{p}^{e} \rtimes \mathbb{Z}_{\frac{p^{e}-1}{2}}$ and $H \cong \mathrm{D}_{q+1}$, such that $T=G H$ and $G \cap H=1$. There exists an involution $g \in T$ such that $\langle H, g\rangle=T$ and $H^{g} \cap H \cong \mathbb{Z}_{2}$. Let $\Gamma=\operatorname{Cos}(T, H, H g H)$. Then, $\Gamma$ is a $T$-arc transitive graph of valency $\frac{q+1}{2}$, and $\Gamma$ has a regular embedding $\mathcal{M}$; see [12. Now $G$ acts regularly on the vertex set $V \Gamma$, and thus $\mathcal{M}$ is a regular Cayley map of the group $G$.

We next give several sporadic examples.

Example 3.9. A group $G \cong \mathrm{A}_{5}$ may be embedded in a group $X \cong \operatorname{PSL}(2,11)$ such that $X=G H$ and $G \cap H=1$ for some cyclic subgroup $H=\langle\rho\rangle \cong \mathbb{Z}_{11}$. By the Atlas [9], any involution $g \in X$ is such that $\langle\rho, g\rangle=X$. Thus, $X=\langle\rho, g\rangle$ is a triangle group and gives rise to a rotary map $\mathcal{M}=\mathcal{M}\langle\rho, g\rangle$ of valency 11; see 12. Since $G$ is a subgroup of $X=\operatorname{PSL}(2,11) \leq \operatorname{Aut} \Gamma$ which is regular on vertices of $\mathcal{M}$, we have that $\mathcal{M}$ is a Cayley map of $G$. If $\mathcal{M}$ is regular, then it follows that Aut $\mathcal{M}=\operatorname{PGL}(2,11)$, which is not possible since $\operatorname{PGL}(2,11)$ has no transitive permutation representation of degree 11 . Thus, $\mathcal{M}$ is chiral.

Example 3.10. A group $G=\mathrm{M}_{10}=\mathrm{A}_{6} \cdot \mathbb{Z}_{2}$ may be embedded in a group $X=\mathrm{M}_{11}$ such that $X=G H$ and $G \cap H=1$, where $H=\langle\rho\rangle \cong \mathbb{Z}_{11}$. Let $g$ be an involution of $X$. It follows from information given in the Atlas 9 that $\langle\rho, g\rangle=X$, and this gives rise to a rotary map $\mathcal{M}=\mathcal{M}\langle\rho, g\rangle$ of valency 11 ; see [12. Further, no involution in $\operatorname{Aut}(X)$ inverts $\rho$. It then follows that $\mathcal{M}$ is a rotary Cayley map of $\mathbf{M}_{10}$. Since an element of order 11 of $X$ is not conjugate in $\operatorname{Aut}(X)=X$ to its inverse, $\mathcal{M}$ is chiral.

Similarly, a group $G=\mathrm{M}_{22}$ may be embedded in a group $X=\mathrm{M}_{23}$ to give rise to a rotary Cayley map $\mathcal{M}\langle\rho, g\rangle$ of $\mathrm{M}_{22}$ (see [12]), where $X=\langle\rho, g\rangle$ with $o(\rho)=23$ and $g^{2}=1$. Since an element of order 23 of $X$ is not conjugate in $\operatorname{Aut}(X)=X$ to its inverse, $\mathcal{M}$ is chiral. So we have the statements:

(1) The group $M_{10}$ has chiral Cayley maps of valency 11.

(2) The Mathieu group $M_{22}$ has chiral Cayley maps of valency 23.

We finally consider symmetric groups and alternating groups. Let $X=\mathrm{S}_{n}$, acting on $\{1,2, \ldots, n\}$. Let $\rho=(123 \ldots n)$, and let $x=(2 n)$. Then, $X=\langle\rho, x\rangle$ gives rise to a rotary map $\mathcal{M}=\mathcal{M}\langle\rho, x\rangle$. Since $X=G H$ where $G=X_{1} \cong \mathrm{S}_{n-1}$ and $H=\langle\rho\rangle$, we have that $\mathcal{M}$ is a Cayley map of $G$. Let

$$
z= \begin{cases}(2, n)(3, n-1) \ldots\left(\frac{n+1}{2}, \frac{n+3}{2}\right), & \text { if } n \text { is odd, } \\ (2, n)(3, n-1) \ldots\left(\frac{n}{2}, \frac{n+4}{2}\right), & \text { if } n \text { is even. }\end{cases}
$$

Then, it is easily shown that $X=\langle x, y, z\rangle$, where $y=\rho z$ and $x^{2}=y^{2}=z^{2}=1$. Thus, $X$ is the automorphism group of the regular map $\mathcal{M}\langle x, y, z\rangle$; see 12. Now let $z^{*}$ be the inner automorphism of $X$ induced by $z$. Then $z^{*}$ inverses $\rho$ and commutes with $x$. Thus, $\mathcal{M}$ is regular and Aut $\mathcal{M}=\left\langle x, \rho, z^{*}\right\rangle \cong X \times \mathbb{Z}_{2}$. It is easily shown that $\mathcal{M}$ is a standard double cover of $\mathcal{M}\langle x, y, z\rangle$.

Lemma 3.11. For each integer $n \geq 5$, the symmetric group $\mathrm{S}_{n-1}$ has a regular Cayley map $\mathcal{M}$ of valency $n$ such that Aut $\mathcal{M} \cong \mathrm{S}_{n} \times \mathbb{Z}_{2}$.

Now we consider the alternating groups. Let $X=\mathrm{A}_{n}$ with $n \geq 5$, acting on $\{1,2, \ldots, n\}$. Let $\rho=(123 \ldots n)$, and let $x=(23)(45)$. Then $X=\langle\rho, x\rangle$, and so gives rise to a rotary map $\mathcal{M}\langle\rho, x\rangle$; see [12]. Let $G=X_{1} \cong \mathrm{A}_{n-1}$ and $H=\langle\rho\rangle$. 
Then $X=G H$ and $G \cap H=1$, and it follows that $G$ acts regularly on vertices of $\mathcal{M}\langle\rho, x\rangle$. Hence, $\mathcal{M}\langle\rho, x\rangle$ is a Cayley map.

Lemma 3.12. For any odd number $n \geq 5$, the alternating group $\mathrm{A}_{n-1}$ has a rotary Cayley map of valency $n$.

\section{Core quotients of Cayley graphs}

In this section, we discuss the first type of operation on Cayley graphs - the core quotient. Then, we prove Theorem 1.3 .

4.1. Taking core quotients. Let $\Gamma=\operatorname{Cay}(G, S)$, and let $X \leq$ Aut $\Gamma$ be an overgroup of $\hat{G}$, that is, $\hat{G}<X \leq$ Aut $\Gamma$. Let $C=$ core $_{X}(\hat{G})$ be the core of $\hat{G}$ in $X$, and let $N \leq C$ such that $N \triangleleft X$. Consider the core quotient $\Gamma_{N}$.

Assume that $\Gamma$ is $X$-edge-transitive. Then, $\Gamma$ is a normal multi-cover of $\Gamma_{N}$. Let $\mathcal{B}$ be the set of $N$-orbits on $V \Gamma_{N}$, and let $K$ be the kernel of the $X$-action on $\mathcal{B}$. Then, $N \triangleleft K, K=N \rtimes K_{1}$, and $\bar{X}:=X / K \leq \operatorname{Aut} \Gamma_{N}$. Moreover, $\Gamma$ is a cover of $\Gamma_{N}$ if and only if $K=N$. The proof of the following lemma is easy; the statement in part (i) was first observed by Praeger [32.

Lemma 4.1. Let $\Gamma=\operatorname{Cay}(G, S)$, and assume that $\hat{G} \leq X \leq$ Aut $\Gamma$ such that $\Gamma$ is $X$-edge transitive. Let $\hat{N} \leq \hat{G}$ be such that $\hat{N} \triangleleft X$, and let $\bar{X}=X / \hat{N}$. Then the following hold:

(i) $\hat{G} / \hat{N}$ is regular on $V \Gamma_{\hat{N}}$, and the core quotient graph $\Gamma_{\hat{N}} \cong \operatorname{Cay}(G / N, S N / N)$;

(ii) for each subgroup $H$ of $\bar{X}$ which is regular on $V \Gamma_{\hat{N}}, \Gamma$ is a Cayley graph of the extension N.H.

As abstract groups, $\hat{G} / C$ is core-free in $X / C$. However, the Cayley graph $\Gamma_{C}$ is not necessarily core-free, since $X / C$ is not necessarily a subgroup of $\Gamma_{C}$. Here is an example.

Example 4.2. Let $X=\mathbb{Z}_{p}^{2} \rtimes \mathrm{S}_{4}<\operatorname{AGL}(2, p)$, where $p$ is an odd prime. Let $H$ be a Sylow 2-subgroup of $X$, so $H \cong \mathrm{D}_{8}$. Set $\Gamma=\operatorname{Cos}\left(X, H, H\left\{g, g^{-1}\right\} H\right)$, where $g$ is an element of $X$ of order 3. Then, $\Gamma$ is an $X$-edge transitive graph. Let $G$ be a Hall $\{3, p\}$-subgroup of $X$. Then, $X=G H$, and $G \cap H=1$. Hence, $\Gamma$ is a Cayley graph of $G$. Now $C:=\operatorname{core}_{X}(G)=\mathbb{Z}_{p}^{2}$, and $X / C \cong \mathrm{S}_{4}$. Further, $\Gamma_{C}$ is a cycle of length 3 , and the kernel $K$ of $X$ acting on the three orbits of $C$ is $K=C \rtimes \mathbb{Z}_{2}^{2}=\mathbb{Z}_{p}^{2} \rtimes \mathbb{Z}_{2}^{2}$. Thus, $\mathrm{S}_{3} \cong X / K \leq \operatorname{Aut} \Gamma_{C}$, and so $\Gamma_{C}$ is a normal Cayley graph of the group $\mathbb{Z}_{3}$. In particular, $\Gamma_{C}$ is not core-free.

However, for the locally primitive case, the state of the matter is different.

Proposition 4.3. Assume that $\Gamma=\operatorname{Cay}(G, S)$ is $X$-locally primitive and such that $\hat{G}<X$. If $N \triangleleft X$ has at least 3 orbits in $V \Gamma$, then $\Gamma$ is a cover of $\Gamma_{N}$, and $X / N \leq$ Aut $\Gamma_{N}$. Further, letting $C=\operatorname{core}_{X}(\hat{G})$, one of the following holds:

(i) $C$ is of index at most 2 in $\hat{G}$, and $\Gamma$ is normal or bi-normal relative to $X$;

(ii) $\Gamma$ is a cover of a core-free Cayley graph.

Proof. If $C$ is transitive on the vertex set $V \Gamma$, then $\Gamma$ is a normal Cayley graph relative to $X$, while if $C$ has two orbits in $V \Gamma$, then $\Gamma$ is a bi-normal Cayley graph relative to $X$. 
Assume that $C$ has at least three orbits on vertices. Then, $\Gamma$ is a cover of the core quotient $\Gamma_{C}$, and $X / C \leq$ Aut $\Gamma_{C}$. Since $\hat{G} / C$ is core-free in $X / C$, we have that $\Gamma_{C}$ is core-free with respect to $X / C$.

For cubic edge-transitive graphs, we have the following conclusion.

Corollary 4.4. Let $\Gamma$ be a $X$-edge-transitive cubic Cayley graph of a group $G$ such that $\hat{G}<X$. Let $C=\operatorname{core}_{X}(\hat{G})$, and $Y=X / C$. Then one of the following holds:

(i) $\hat{G}$ is normal in $X$, and $X_{1}=\mathbb{Z}_{3}$ or $\mathrm{S}_{3}$;

(ii) $\hat{G}$ is bi-normal in $X$, and $X_{1}=\mathbb{Z}_{3}$;

(iii) $\Gamma$ is a cover of $\Gamma_{C}, \Gamma_{C}$ is a $Y$-edge-transitive Cayley graph of $G / C$, and $Y$ is isomorphic to a subgroup of $\mathrm{S}_{48}$ and $Y$ has an exact factorisation with one factor isomorphic to $\mathrm{S}_{3}, \mathrm{~S}_{3} \times \mathbb{Z}_{2}, \mathrm{~S}_{4}$ or $\mathrm{S}_{4} \times \mathbb{Z}_{2}$.

Proof. If $\hat{G}$ is normal in $X$, then since $\Gamma$ is connected, $X_{1}$ is faithful on $S$, and hence $X_{1}=\mathbb{Z}_{3}$ or $\mathrm{S}_{3}$. Assume that $\hat{G}$ is bi-normal in $X$. Then, $\Gamma$ is bipartite, with parts $\Delta_{1}$ and $\Delta_{2}$ say. Let $X^{+}=X_{\Delta_{1}}=X_{\Delta_{2}}$. Then $X_{1}=X_{1}^{+}$. By [22, Corollary 2.7], $X_{1}$ is faithful on $S$, and hence $X_{1}=\mathbb{Z}_{3}$ or $S_{3}$. Since $X$ has no normal regular subgroup, we conclude that $X / C \cong X_{1} \cdot \mathbb{Z}_{2} \neq X_{1} \times \mathbb{Z}_{2}$. It follows that $X_{1} \cong \mathbb{Z}_{3}$.

Assume that $C$ has at least three orbits in $V \Gamma$. Then, by Lemma 4.1] and Proposition 4.3. we have that $\Gamma_{C}$ is a $Y$-edge-transitive Cayley graph of a group isomorphic to $\hat{G} / C$, and $\Gamma$ is a cover of $\Gamma_{C}$. Further, by the well-known theorem of Tutte (see [2]), we obtain the statement in part (iii).

4.2. Locally primitive holomorph Cayley graphs. Here we first characterize locally primitive central Cayley graphs.

Lemma 4.5. Let $\Gamma=\operatorname{Cay}(G, S)$ be a connected Cayley graph of valency at least 3 , and let $X=\hat{G} \circ \tilde{H} \leq \operatorname{Aut} \Gamma$ for some subgroup $H \leq G$. Suppose that $X_{\mathbf{1}}$ acts primitively on $S$. Then $S=s^{H}$, where $s$ is an involution such that $\mathbf{C}_{H}(s)$ is a maximal subgroup of $H$, and $G$ has a normal subgroup $N$ such that $X_{\mathbf{1}} \cap \hat{N}=1, \Gamma_{\hat{N}}$ is a Cayley graph of $G / N$ and $\Gamma$ is a normal cover of $\Gamma_{\hat{N}}$. Further, $G / N=T, T . \mathbb{Z}_{2}$ or $T^{2} \cdot \mathbb{Z}_{2}$, where $T$ is a simple group, and one of the following holds:

(i) $G / N=T$ is non-abelian;

(ii) $\Gamma$ is bipartite, $G / N=T \cdot \mathbb{Z}_{2}$ or $T^{2} \cdot \mathbb{Z}_{2}$, and $T$ is non-abelian;

(iii) $\Gamma_{\hat{N}} \cong \mathrm{K}_{p, p}, T=\mathbb{Z}_{p}$ with $p$ odd prime and $G / N=\mathrm{D}_{2 p}$.

Proof. The group $X_{1}$ is a primitive permutation group on $S$. It then follows that $S=s^{H}$ for some involution $s$. The stabiliser of $s$ for this $X_{1}$ action on $S$ is the arc stabiliser $X_{\mathbf{1} s}$, which consists of the inner automorphisms of $G$ induced by elements of $H$ fixing $s$, that is, the elements of $\mathbf{C}_{H}(s)$. Thus, as $X_{1 s}$ is a maximal subgroup of $X_{\mathbf{1}}, \mathbf{C}_{H}(s)$ is a maximal subgroup of $H$.

Let $N$ be a maximal normal subgroup of $G$ of index at least 3 . Since $X=\hat{G} \circ \tilde{H}$, each element of $\tilde{H}$ centralizes $\hat{G}$, and so $\hat{N}$ is a normal subgroup of $X$. As $\hat{G}$ is regular on $V \Gamma$, the subgroup $\hat{N}$ has $|\hat{G} / \hat{N}|$ orbits in $V \Gamma$. By Proposition 4.3, the normal quotient $\Gamma_{\hat{N}}$ is $\bar{X}$-locally primitive where $\bar{X}=X / \hat{N}$, and $\Gamma$ is a cover of $\Gamma_{\hat{N}}$. By Lemma 4.1, $\Gamma_{\hat{N}}$ is a Cayley graph of $G / N$.

By the choice of $N$, each non-trivial minimal normal subgroup of $G / N$ has index at most 2. It follows that $G / N=T, T \cdot \mathbb{Z}_{2}$ or $(T \times T) \cdot \mathbb{Z}_{2}$, where $T$ is simple. If $G / N=T$, then by Lemma 2.2 (iii), we conclude that $T$ is non-abelian. 
Assume that $G / N=T \cdot \mathbb{Z}_{2}$ or $(T \times T) \cdot \mathbb{Z}_{2}$. Since $\hat{G} / \hat{N}$ is regular on $V \Gamma_{\hat{N}}$, the socle $\operatorname{soc}(\hat{G} / \hat{N})$ has exactly two orbits in $V \Gamma_{\hat{N}}$. It follows that $\Gamma_{\hat{N}}$ is bipartite, and hence $\Gamma$ is bipartite. Assume further that $T=\mathbb{Z}_{p}$ for some prime $p$. If $G / N=(T \times T) \cdot \mathbb{Z}_{2}$, then $T \times T$ is not a minimal normal subgroup of $G / N$, which is a contradiction. Thus, $G / N=T \cdot \mathbb{Z}_{2}=\mathbb{Z}_{p} \cdot \mathbb{Z}_{2}$. Since $\Gamma_{\hat{N}}$ has valency at least 3 , it follows that $G / N$ is non-abelian. Hence, $G / N=\mathrm{D}_{2 p}$. Now $\Gamma_{\hat{N}}$ has valency $p$, and so $\Gamma_{\hat{N}}=\mathrm{K}_{p, p}$.

We now give a technical lemma, which tells us that if a group $X$ acts on a graph edge-transitively, then the commutator group $X^{\prime}$ is 'large'.

Lemma 4.6. Let $\Gamma$ be a connected $X$-edge transitive graph. Then, the the normal quotient $\Gamma_{X^{\prime}}$ is a cycle. If further $X$ is transitive on the arcs of $\Gamma$, then $X^{\prime}$ has at most two orbits in $V \Gamma$. If in addition $\Gamma=\operatorname{Cay}(G, S)$ and $X=\hat{G} \circ \tilde{G}$, then $G / G^{\prime}=1$ or $\mathbb{Z}_{2}$.

Proof. If $X^{\prime}$ is transitive on $V \Gamma$, then we are done. Assume that $X^{\prime}$ is intransitive on $V \Gamma$. Let $\mathcal{B}$ be the set of $X^{\prime}$-orbits in $V \Gamma$, and let $K$ be the kernel of $X$ acting on $\mathcal{B}$. Then, $X^{\prime} \leq K \triangleleft X, X / K \leq$ Aut $\Gamma_{X^{\prime}}$, and $\Gamma_{X^{\prime}}$ is $(X / K)$-edge-transitive. Now $X / K$ is abelian, and hence $X / K$ is regular on both $V \Gamma_{X^{\prime}}$ and $E \Gamma_{X^{\prime}}$. In particular, $\left|V \Gamma_{X^{\prime}}\right|=\left|E \Gamma_{X^{\prime}}\right|$. Since $\Gamma$ is connected, $\Gamma_{X^{\prime}}$ is connected, and thus $\Gamma_{X^{\prime}}$ is a cycle.

Further, assume that $\Gamma$ is $X$-arc-transitive. Then, $\Gamma_{X^{\prime}}$ is $(X / K)$-arc-transitive. It follows that $\Gamma_{X^{\prime}} \cong \mathrm{K}_{2}$, and $X^{\prime}$ has two orbits in $V \Gamma$.

Assume that $\Gamma=\operatorname{Cay}(G, S)$ and $X=\hat{G} \circ \tilde{G}$. Then, the commutator subgroup $\hat{G}^{\prime}$ is normal in $X$, and the induced action of $X$ on $V \Gamma_{\hat{G}^{\prime}}$ is abelian. Hence, $\hat{G}^{\prime}$ has at most two orbits in $V \Gamma$, and so $G / G^{\prime}=1$ or $\mathbb{Z}_{2}$.

Applying Lemma 4.5 to a Cayley graph $\Gamma=\operatorname{Cay}(G, S)$ with $H=G$, we have the following result.

Lemma 4.7. Let $G$ be a group with $\mathbf{Z}(G)=1$, and let $\Gamma=\operatorname{Cay}(G, S)$ be connected. Let $X=\hat{G} \times \tilde{G}$. Assume that $\Gamma$ is $X$-locally primitive. Then, $S=s^{G}$ for some involution s such that $\mathbf{C}_{G}(s)$ is a maximal subgroup of $G$ and one of the following holds:

(i) $G=\mathrm{D}_{2 p}$ with $p$ prime, and $\Gamma \cong \mathrm{K}_{p, p}$;

(ii) $G=T$ is a non-abelian simple group;

(iii) $G=T \rtimes\langle s\rangle$ with $T$ non-abelian simple, and $\mathbf{C}_{G}(s)=\mathbf{C}_{T}(s) \times\langle s\rangle$;

(iv) $G=T^{2} \rtimes\langle s\rangle$ with $T$ non-abelian simple, and $\mathbf{C}_{G}(s)=T \times\langle s\rangle$.

Proof. By Lemma 4.5 there exists an involution $s$ such that $S=s^{G}$ and $\mathbf{C}_{G}(s)$ is a maximal subgroup of $G$. Since $\Gamma$ is connected, $X_{1} \cong G$ acts faithfully on $S$.

Suppose that $G$ has a normal subgroup $N$ of index at least 3. Since $\hat{G}$ is regular on the vertex set $V$ of $\Gamma, \hat{N}$ has at least 3-orbits in $V$. Since $\Gamma$ is $X$-locally primitive, $(\hat{G} / \hat{N}) \times \tilde{G} \cong X / \tilde{N} \leq$ Aut $\Gamma_{\hat{N}}, \Gamma_{\hat{N}}$ is $(X / \hat{N})$-locally primitive, and $\Gamma$ is a cover of $\Gamma_{\hat{N}}$. As $\hat{G} / \hat{N}$ is a regular subgroup of $\operatorname{Sym}\left(V_{\hat{N}}\right)$, we have $\mathbf{C}_{\operatorname{Sym}\left(V_{\hat{N}}\right)}(\hat{G} / \hat{N}) \cong \hat{G} / \hat{N}$. However, $\tilde{G}=\mathbf{C}_{X / \hat{N}}(\hat{G} / \hat{N}) \leq \mathbf{C}_{\operatorname{Sym}\left(V_{\hat{N}}\right)}(\hat{G} / \hat{N}) \cong \hat{G} / \hat{N}$, which is a contradiction. Thus, each non-trivial normal subgroup of $G$ has index at most 2 . It follows that $G=T, T \cdot \mathbb{Z}_{2}$ or $T^{2} \cdot \mathbb{Z}_{2}$, where $T$ is a simple group.

If $G=T$, then $X=\hat{G} \times \tilde{G} \cong T \times T \leq \operatorname{Sym}(T)$, and $T$ is non-abelian.

Assume then that $G=T \cdot \mathbb{Z}_{2}$. Since $\Gamma$ is connected, we have $\left\langle s^{G}\right\rangle=G$, and so $s \notin T$. Hence, $G=T \rtimes\langle s\rangle$, and $\mathbf{C}_{G}(s)=\mathbf{C}_{T}(s) \times\langle s\rangle$. In particular, if $T=\mathbb{Z}_{p}$ with $p$ prime, then $p \geq 3, G=\mathrm{D}_{2 p}$, and $\mathbf{C}_{G}(s)=\langle s\rangle$. 
We now assume that $G=T^{2} \cdot \mathbb{Z}_{2}=\left(T_{1} \times T_{2}\right) \cdot \mathbb{Z}_{2}$, where $T_{1} \cong T_{2} \cong T$. Then, $T_{1} \times T_{2}$ is the unique minimal normal subgroup of $G$, and hence $T_{i}$ is non-abelian simple. Since $\Gamma$ is connected, $\left\langle s^{G}\right\rangle=G$, and so $s \notin T^{2}$. Hence, $G=\left(T_{1} \times T_{2}\right) \rtimes\langle s\rangle$, and $s$ interchanges $T_{1}$ and $T_{2}$. Let $D=\left\langle\left(t_{1}, t_{1}^{s}\right) \mid t_{1} \in T_{1}\right\rangle$. Then, $D \cong T$ and $\mathbf{C}_{G}(s)=\langle D, s\rangle=D \times\langle s\rangle$.

4.3. Proof of Theorem 1.3. To complete the proof of Theorem 1.3, we need a property of point stabilisers of a 2-transitive permutation group. Inspecting the classification of finite 2-transitive permutation groups (see [4), we conclude the following property.

Lemma 4.8. Let $X$ be an almost simple 2-transitive permutation group on $\Omega$ with socle $T$. Then, neither $\mathbf{Z}\left(T_{\omega}\right)$ nor $\mathbf{Z}\left(X_{\omega}\right)$ contains an involution, for any $\omega \in \Omega$.

Now we are ready to prove Theorem 1.3 .

Proof of Theorem 1.3. Let $\Gamma=\operatorname{Cay}(G, S)$ be a holomorph Cayley graph, and let $X=\hat{G} \circ \tilde{G}$. Then, $X=\hat{G} \rtimes X_{1} \leq$ Aut $\Gamma$, and $X_{1} \cong \operatorname{lnn}(G) \cong G / \mathbf{Z}(G)$. Assume further that $\Gamma$ is $X$-locally primitive. Then, by Lemma 4.5, $S=s^{G}$ for some involution $s$, and $\mathbf{C}_{G}(s)$ is a maximal subgroup of $G$.

It is known that $\hat{G} \cap \tilde{G}=\mathbf{Z}(\hat{G})=\mathbf{Z}(\tilde{G})$. Let $Z=\mathbf{Z}(\hat{G})=\mathbf{Z}(\tilde{G})$, and let $\bar{X}=X / Z$. Then, $\bar{X}=(\hat{G} / Z) \times(\tilde{G} / Z), \hat{G} / Z$ is regular on the vertex set of the quotient graph $\Gamma_{Z}$, and $\bar{X} \leq$ Aut $\Gamma_{Z}$. Thus, $\mathbf{Z}(\hat{G} / Z)=1, \Gamma_{Z}$ is a Cayley graph of a group $H$, where $H \cong \hat{G} / Z$, and $\Gamma_{Z}$ is $\bar{X}$-locally primitive. By Lemma 4.7, we have that either $H=\mathrm{D}_{2 p}$ with $p$ odd prime, or $H=T, T . \mathbb{Z}_{2}$ or $T^{2} . \mathbb{Z}_{2}$, where $T$ is a non-abelian simple group.

We first suppose that $H=\mathrm{D}_{2 p}$ with $p$ odd prime. Then, $G=Z . H=Z . \mathrm{D}_{2 p}=$ $Z_{\{2, p\}^{\prime}} \times\left(Z_{\{2, p\}} . D_{2 p}\right)$, where $Z_{\{2, p\}^{\prime}}$ is a Hall $\{2, p\}^{\prime}$-subgroup of $Z$ and is abelian. By Lemma 4.6] we have that $Z_{\{2, p\}^{\prime}}=1$. For the Sylow 2-subgroup $Z_{2}$ and the Sylow $p$-subgroup $Z_{p}$ of $Z$, we have that $G / Z_{p}=Z_{2} \cdot \mathrm{D}_{2 p}=\mathbb{Z}_{p} \rtimes\left(Z_{2} \cdot \mathbb{Z}_{2}\right)=\mathbb{Z}_{p} \rtimes$ $\left(Z_{2} \times\langle s\rangle\right)=\left(\mathbb{Z}_{p} \rtimes\langle s\rangle\right) \times Z_{2}$. By Lemma 4.6, we have $Z_{2}=1$ or $\mathbb{Z}_{2}$. If $Z_{2}=\mathbb{Z}_{2}$, then $G=\mathrm{D}_{2 p} \times \mathbb{Z}_{2}$; however, $G$ has no conjugacy class generating $G$, which is a contradiction. Thus, $Z_{2}=1$, and $Z$ is a $p$-group which is abelian. Suppose that $Z \neq 1$, and let $P<Z$ be such that $Z / P=\mathbb{Z}_{p}$. Then, $G / P=\mathbb{Z}_{p}$. $D_{2 p}=\left(\mathbb{Z}_{p} . \mathbb{Z}_{p}\right) \rtimes \mathbb{Z}_{2}$. It follows that $G / P=\mathbb{Z}_{p} \times \mathrm{D}_{2 p}$, which is not possible by Lemma 4.6. Hence, $Z=1$ and $G=H=\mathrm{D}_{2 p}$, as in part (i) of Theorem 1.3 .

Suppose then that $G / \mathbf{Z}(G)=H=T$ or $T . \mathbb{Z}_{2}$, where $T$ is non-abelian simple. As $G / G^{\prime}=1$ or $\mathbb{Z}_{2}$, we conclude that $G$ is an almost quasi-simple group, as in part (ii) or (iii) of Theorem 1.3 .

Suppose finally that $G / \mathbf{Z}(G)=T^{2} \cdot \mathbb{Z}_{2}=\left(T_{1} \times T_{2}\right) \cdot \mathbb{Z}_{2}$. Then, $s$ interchanges $T_{1}$ and $T_{2}$. Let $Y$ be the full preimage of $T^{2}$ under $G \rightarrow G / \mathbf{Z}(G)$. Then, the commutator subgroup $Y^{\prime}$ is normal in $G$. Thus, $Y^{\prime}$ has index at most 2 in $G$, and so $Y^{\prime}=Y$. Let $U_{i}=\mathbf{Z}(G) \cdot T_{i}$, where $i=1$ or 2 . Then, $s$ interchanges $U_{1}$ and $U_{2}$, and so interchanges the commutator subgroups $U_{1}^{\prime}$ and $U_{2}^{\prime}$. Further, $U_{1}^{\prime} U_{2}^{\prime}$ char $U_{1} U_{2}=Y \triangleleft G$. If $U_{1}^{\prime} \neq U_{1}$, then $U_{1}^{\prime} U_{2}^{\prime}$ has index greater than 2 in $G$, which is a contradiction. Thus, $U_{1}^{\prime}=U_{1}$ and $U_{2}^{\prime}=U_{2}$, and so both $U_{1}$ and $U_{2}$ are quasi-simple. We obtain that $Y=U_{1} \circ U_{2}$, and $G=\left(U_{1} \circ U_{2}\right) \rtimes\langle s\rangle$. Since $s$ interchanges $U_{1}$ and $U_{2}$, the diagonal subgroup $\left\langle\left(u, u^{s}\right) \mid u \in U_{1}\right\rangle$ is isomorphic to $U_{1}$ and centralised by $s$, as in part (iv) of Theorem 1.3 . 
Finally, assume that $\Gamma$ is $(X, 2)$-arc transitive. Suppose that $G$ is insoluble, that is, one of parts (ii)-(iv) of Theorem 1.3 occurs (just established above). By Corollary 2.5. the stabiliser $X_{\mathbf{1} s} \cong \mathbf{C}_{G}(s) / \mathbf{Z}(G)$. Since $\Gamma$ is connected, $s \notin \mathbf{Z}(G)$, and hence the image of $s$ under $\mathbf{C}_{G}(s) \rightarrow \mathbf{C}_{G}(s) / \mathbf{Z}(G)$ is an involution, which is a contradiction to Lemma 4.8. Thus, $G \cong \mathrm{D}_{2 p}$, and $\Gamma \cong \mathrm{K}_{p, p}$. Then, $X_{1} \cong \mathrm{D}_{2 p}$ has a 2 -transitive permutation representation if and only if $p=3$. Therefore, $\mathrm{K}_{3,3}$ is the only holomorph 2-arc-transitive Cayley graph. This completes the proof of Theorem 1.3 .

At last, we give a short proof for the following theorem of Praeger [31] regarding quasiprimitive permutation 2-arc transitive graphs. This proof is independent of O'Nan-Scott theorem for quasiprimitive permutation groups.

Theorem 4.9. (Praeger 1992) Let $\Gamma$ be an $(X, 2)$-arc-transitive graph with vertex set $V$, and assume that $X$ is quasiprimitive on $V$. Then, either $\operatorname{soc}(X)$ is regular on $V$, or $\operatorname{soc}(X)$ has no normal subgroup which is regular on $V$; so in terms of O'Nan-Scott theorem, $X$ is of type HA, TW, AS or PA.

Proof. Suppose that $N:=\operatorname{soc}(X)$ has a proper normal subgroup which is regular on $V$. Then, $N$ is non-abelian and may be written as $N=M \times L$, where $M=T^{m}$ is regular on $V$ and $L=T^{l}$ for some positive integers $m$ and $l$. For $v \in V$, since $X_{v}^{\Gamma(v)}$ is 2-transitive and $T^{l}=L \cong N_{v} \cong N_{v}^{\Gamma(v)} \triangleleft X_{v}^{\Gamma(v)}$, it follows that $L \cong N_{v}^{\Gamma(v)} \cong T$. In particular, $N_{v}$ is primitive on $\Gamma(v)$. Let $H \triangleleft M$ be such that $H \cong T^{m-1}$. Then, $T^{2} \cong N / H \leq$ Aut $\Gamma_{H}$, and the normal quotient $\Sigma=\Gamma_{H}$ is a Cayley graph of the group $M / H \cong T$.

Write $G=M / H$, and $Y=N / H$. Then, $Y=\hat{G} \times \tilde{G}, Y_{1} \cong G \cong T$, and $\Sigma=\operatorname{Cay}(G, S)$ for some subset $S$. Further, $Y_{1}^{S} \cong N_{v}^{\Gamma(v)} \triangleleft X_{v}^{\Gamma(v)}$; in particular, $Y_{1}$ is primitive on $S$. By Corollary 2.5. $S=s^{G}$ for some involution $s$, and $Y_{1 s} \cong$ $\mathbf{C}_{G}(s)$. So, the center $\mathbf{Z}\left(Y_{\mathbf{1} s}\right)$ contains an involution $s$, which is a contradiction to Lemma 4.8. This completes the proof of the theorem.

\section{Normal QUOTIENTS}

A normal quotient of a Cayley graph need not be a Cayley graph. This property allows us to work out a potential method for constructing vertex-transitive nonCayley graphs, as seen in Proposition 5.1. On the other hand, for a certain type of Cayley graph, its normal quotient is also a Cayley graph, which enables us to characterise the Cayley graph; see Proposition 5.5.

5.1. Normal quotient are not necessarily Cayley graphs. Here we give a characterisation result about cubic edge-transitive Cayley graphs.

Proposition 5.1. Let $G$ be a non-abelian simple group such that $G=\langle a, b\rangle$, where $a$ is an involution and $b$ has order 3 . Let $S=\left\{a, a^{b}, a^{b^{2}}\right\}$, and let $\Gamma=\operatorname{Cay}(G, S)$. Let $\Sigma=\operatorname{Cos}(G,\langle b\rangle,\langle b\rangle a\langle b\rangle)$. Then, both graphs $\Gamma$ and $\Sigma$ are connected cubic arctransitive graphs, and further,

(i) $\Gamma$ is a normal cover of $\Sigma$;

(ii) if $G$ has no transitive permutation representation of degree $n \leq 15$, then $\Sigma$ is a non-Cayley graph. 
Proof. Since $G=\langle S\rangle=\langle a, b\rangle$, both graphs $\Gamma$ and $\Sigma$ are connected cubic graphs. Let $\rho$ be the inner automorphism of $\Gamma$ induced by $b$. By Lemma 2.1 $\rho \in \operatorname{Aut}(G, S)$ is an automorphism of the graph $\Gamma$, and

$$
X:=\langle\hat{G}, \rho\rangle=\hat{G} \rtimes\langle\rho\rangle=\hat{G} \times C \leq \operatorname{Aut} \Gamma,
$$

where $C=\langle\tilde{b}\rangle \cong \mathbb{Z}_{3}$. In particular, $\Gamma$ is $X$-arc transitive.

Thus, the normal quotient $\Gamma_{C}$ is $(X / C)$-arc transitive and cubic. It is easily shown that the quotient graph $\Gamma_{C}$ is isomorphic to the coset graph $\Sigma$, by Proposition 5.3. Thus, we only need to prove that if $\Sigma$ is a Cayley graph, then $G \leq \mathrm{S}_{15}$.

For convenience, denote by $V$ the vertex set of $\Sigma$, and by $G$ the group $\hat{G}$. Then, $|V|=|G| / 3$, and since $|Y| /|V|$ divides 48 , we have that $|Y: G|$ divides $2^{4}$.

Assume first that $G$ is not normal in $Y$. Then, $Y$ acts faithfully on $[Y: G]$, which is of degree dividing $2^{4}$. Since $G$ is non-abelian simple, it follows that $[Y: G]$ has size 8 or 16. In particular, $G$ is a subgroup of $\mathrm{S}_{15}$. Therefore, if $G \not \leq \mathrm{S}_{15}$, then $G$ is normal in $Y$.

Assume now that $G$ is normal in $Y$. Then, for a vertex $v \in V$, we have $\mathbb{Z}_{3} \cong$ $G_{v} \triangleleft Y_{v}$. Thus, $Y_{v}=\mathrm{S}_{3}$ or $\mathrm{D}_{12}, \Sigma$ is 2 - or 3 -arc transitive, and $Y / G \cong \mathbb{Z}_{2}$ or $\mathbb{Z}_{2}^{2}$, respectively. Suppose that $Q<Y$ is regular on $V$. Then, $Z:=Q G \leq Y$ and $\Sigma$ is $Z$-arc transitive. Thus, by the well-known theorem of Tutte, $Z_{v}=\mathbb{Z}_{3}, \mathrm{~S}_{3}$ or $\mathrm{D}_{12}$. Since $|Z|=|Q G|=|Q| \cdot \frac{|G|}{|Q \cap G|}$, we have $\frac{|G|}{|Q \cap G|}=3,6$ or 12 . We conclude that $G$ has a transitive permutation representation of degree 6 or 12 . Hence, $G=\mathrm{A}_{5}, \mathrm{~A}_{6}$, $\operatorname{PSL}(2,11), \mathrm{M}_{11}, \mathrm{M}_{12}$, or $\mathrm{A}_{12}$. In particular, $G<\mathrm{S}_{15}$.

Proposition 5.1 provides a potential method for constructing vertex-transitive graphs that are not Cayley graphs.

Example 5.2. Let $G=\mathrm{A}_{n}$, where $n \geq 16$. Let $G=\langle a, b\rangle$, where $a^{2}=1=b^{3}$. Let $\Sigma=\operatorname{Cos}(G,\langle b\rangle,\langle b\rangle a\langle b\rangle)$. Then, $\Sigma$ is a connected cubic arc-transitive graph and is not a Cayley graph.

Remark. By the result of [26], except for $\operatorname{PSp}\left(4,2^{d}\right), \operatorname{PSU}\left(3,3^{d}\right), \mathrm{Sz}\left(2^{d}\right)$ and a finite list of other groups, every finite non-abelian simple group may be generated by an element of order 3 and an involution. Therefore, except for $\operatorname{PSp}\left(4,2^{d}\right), \operatorname{PSU}\left(3,3^{d}\right)$, $\mathrm{Sz}\left(2^{d}\right)$ and a finite list of other groups, every finite non-abelian simple group is an automorphism group of a cubic arc transitive graph which is not a Cayley graph.

Next we turn our attention to the general case. It will be shown that each vertextransitive, half-arc-transitive, and arc-transitive graph is a normal quotient of a vertex-transitive, half-arc-transitive, and arc-transitive Cayley graph, respectively.

Let $X$ be a transitive permutation group on a set $\Omega$. Let $C \leq \mathbf{C}_{\mathrm{Sym}(\Omega)}(X)$, and let $Y=\langle X, C\rangle$. Then, either $X$ is regular on $\Omega$, or $C$ is intransitive on $\Omega$; see 4 . If $C$ is intransitive, then the $C$-orbits on $\Omega$ form a block system of imprimitivity of $Y$, as well as of $X$. This quotient of $Y$ induced by $C$ is said to be a central quotient of $X$. The following proposition describes a relation between edge-transitive graphs and edge-transitive Cayley graphs.

Proposition 5.3. Let $G$ be a finite group, and let $H$ be a core-free subgroup of $G$ and $s \in G$. Set

$$
\Gamma=\operatorname{Cay}\left(G,\left\{s, s^{-1}\right\}^{H}\right) \text { and } \Sigma=\operatorname{Cos}\left(G, H, H\left\{s, s^{-1}\right\} H\right) .
$$


Then, $\Gamma$ is a central edge-transitive Cayley graph of $G$, and further,

(i) $\Sigma$ is a $\hat{G}$-central quotient of $\Gamma$;

(ii) if $\Gamma$ is a cover of $\Sigma$, then $s$ centralizes the arc stabilizer $G_{\alpha \beta}$, where $\alpha$ and $\beta$ denote the vertices $H$ and $H^{s}$, respectively.

Proof. It follows from definition that $X:=\hat{G} \circ \tilde{H} \leq$ Aut $\Gamma$, and $X$ is transitive on the edge set of $\Gamma$. Hence, $\Gamma$ is a central edge-transitive Cayley graph of $G$. Since $H$ is core-free, we have that $\hat{G} \cap \tilde{H}=1$, and so $X=\hat{G} \times C$, where $C=\tilde{H}$. Thus, the vertex stabilizer $X_{1}=\langle(\hat{h}, \tilde{h}) \mid h \in H\rangle \cong H$. Now $X / C \cong G$, and $\left(X_{1} C\right) / C \cong H$. Let $\overline{\mathbf{1}}$ be the vertex of $\Gamma_{C}$ which is the orbit of $C$ containing 1 . Then, the vertex stabilizer of $\overline{\mathbf{1}}$ in $X / C$ is $\left(X_{1} C\right) / C$. We may identify $X / C$ with $G$ and $\left(X_{1} C\right) / C$ with $H$. It then follows that the quotient $\Gamma_{C}$ is isomorphic to $\Sigma$, and $X / C \cong G$ is transitive on the vertices and the edges of $\Sigma$.

Assume further that $\Gamma$ is a cover of $\Sigma$. Then, $\operatorname{val}(\Sigma)=\operatorname{val}(\Gamma)$. Suppose first that $\Gamma$ is $X$-arc transitive. Then, $s^{h}=s^{-1}$ for some $h \in H$, and so $\left\{s, s^{-1}\right\}^{H}=s^{H}$. Hence, $\operatorname{val}(\Gamma)=\left|s^{H}\right|=\left|H: \mathbf{C}_{H}(s)\right|, H s H=H s^{-1} H$, and $H\left\{s, s^{-1}\right\} H=H s H$. Further, $\operatorname{val}(\Sigma)=\left|H: H \cap H^{s}\right|$. Since $\operatorname{val}(\Gamma)=\operatorname{val}(\Sigma)$, it follows that $\mathbf{C}_{H}(s)=$ $H \cap H^{s}=G_{\alpha \beta}$. So $s$ centralizes $G_{\alpha \beta}$.

Suppose now that $\Gamma$ is not $X$-arc transitive. Then, $s$ is not conjugate under $H$ to $s^{-1}$, and so

$$
\begin{aligned}
\operatorname{val}(\Gamma) & =\left|\left\{s, s^{-1}\right\}^{H}\right|=\left|s^{H}\right|+\left|\left(s^{-1}\right)^{H}\right| \\
& =\left|H: \mathbf{C}_{H}(s)\right|+\left|H: \mathbf{C}_{H}\left(s^{-1}\right)\right|=2\left|H: \mathbf{C}_{H}(s)\right| .
\end{aligned}
$$

Since $\mathbf{C}_{H}(s) \leq H \cap H^{s}$, we have $\left|H: \mathbf{C}_{H}(s)\right| \geq\left|H: H \cap H^{s}\right|$. On the other hand,

$$
2\left|H: \mathbf{C}_{H}(s)\right|=\operatorname{val}(\Gamma)=\operatorname{val}(\Sigma)=\left|H: H \cap H^{s}\right|+\left|H: H \cap H^{s^{-1}}\right| .
$$

It follows that $H s H \neq H s^{-1} H$ and $\mathbf{C}_{H}(s)=H \cap H^{s}=G_{\alpha \beta}$. So again $s$ centralizes $G_{\alpha \beta}$. This completes the proof of the proposition.

5.2. Rotary Cayley maps of abelian groups. Here we apply the technique of taking a normal quotient to give a characterisation of rotary Cayley maps of abelian groups. It is known that all complete graphs of prime-power order admit rotary embeddings as Cayley maps of elementary abelian groups, only one (the tetrahedron) of which is regular; see [1] or [3. Infinitely many regular Cayley maps of abelian groups are given in the following example.

Example 5.4. Let $\Gamma=\mathbf{Q}_{d}$, the hypercube of dimension $d$. As described in Example 2.3. Aut $\Gamma \cong \mathbb{Z}_{2}^{d} . S_{d}$, and in particular, $\Gamma$ is a Cayley graph of a group $G \cong \mathbb{Z}_{2}^{d}$. There is a subgroup $X \leq$ Aut $\Gamma$ such that $X \cong \mathbb{Z}_{2}^{d}$. $\mathrm{D}_{2 d}$ and $X$ acts arc-transitively on $\Gamma$. It follows that $\Gamma$ has a regular orientable embedding which gives rise to a regular map $\mathcal{M}$. Now $\hat{G}$ acts regular on the vertices of $\mathcal{M}$, and $\hat{G}$ is normal in $X$. So $\mathcal{M}$ is a balanced regular Cayley map.

The next proposition gives a characterisation of rotary Cayley maps of abelian groups. It particularly shows that the chirality of Cayley maps given in Proposition 3.6 belongs to all cyclic rotary maps of odd order. (A Cayley map of a cyclic group is called a cyclic map.) A group is called di-abelian if it is a split extension of an abelian group by the cyclic group $\mathbb{Z}_{2}$. 
Proposition 5.5. Let $\mathcal{M}$ be a rotary orientable Cayley map of an abelian group. Then, either

(1) $\mathcal{M}$ is a balanced Cayley map of an abelian group, or

(2) $\mathcal{M}$ is bipartite and is not balanced with respect to any abelian group; further, one of the following holds:

(i) $\mathcal{M}$ is a balanced Cayley map of a di-abelian group;

(ii) $\mathcal{M}=\mathcal{M}^{\prime}\left[\overline{\mathrm{K}}_{b}\right]$, where $\mathcal{M}^{\prime}$ is a balanced Cayley map of an abelian group or a di-abelian group.

In particular, if $\mathcal{M}$ has odd number of vertices, then $\mathcal{M}$ is a balanced Cayley map of an abelian group; if $\mathcal{M}$ is cyclic of odd order, then $\mathcal{M}$ is chiral.

Proof. Let $G$ be an abelian base group of $\mathcal{M}$, and let $X=$ Aut $\mathcal{M}$. Then $\hat{G} \leq X$ and $X=\hat{G} X_{1}$, such that $X_{1}$ is cyclic. In particular, $X$ is a product of two abelian subgroups $\hat{G}$ and $X_{1}$. The well-known result of Ito [15] tells us that $X^{\prime}$ is abelian. On the other hand, by Lemma 4.6, $X^{\prime}$ has at most two orbits in $V$.

If $X^{\prime}$ is transitive on $V$, then $X^{\prime}$ is regular on $V$. Thus, $\mathcal{M}$ may be represented as a Cayley map of an abelian group $F$ such that $X^{\prime}=\hat{F}$. By [34], $\mathcal{M}$ is a balanced rotary Cayley map of the base group $F$, as in part (1).

Assume next that $X^{\prime}$ has two orbits $\Delta$ and $\Delta^{\prime}$ in $V \Gamma$. Take an $\operatorname{arc}(\alpha, \beta)$ of $\Gamma$. Since $\Gamma$ is $X$-arc transitive, there exists an involution $g \in X$ interchanging $\alpha$ and $\beta$. Thus, the subgroup $Y=\left\langle X^{\prime}, g\right\rangle=X^{\prime} \cdot \mathbb{Z}_{2}$ is di-abelian and transitive on $V$.

If $X^{\prime}$ is faithful on $\Delta$, then $X^{\prime}$ is faithful on $\Delta^{\prime}$, and $X^{\prime}$ is regular on both $\Delta$ and $\Delta^{\prime}$. It follows that $Y$ is regular on $V \Gamma$, and thus $\mathcal{M}$ may be represented as a Cayley map of $Y$. Since $X / X^{\prime}$ is abelian, $Y / X^{\prime}$ is normal in $X / X^{\prime}$, and so $Y$ is normal in $X$. Hence, by [34, $\mathcal{M}$ is a balanced rotary Cayley map of $Y$, as in part (2)(i).

Suppose now that $X^{\prime}$ is unfaithful on $\Delta$. Let $K$ be the kernel of $X^{\prime}$ acting on $\Delta$. Then, $K^{g}$ is the kernel of $X^{\prime}$ acting on $\Delta^{\prime}$. Since $X$ acts on $V$ faithfully, we have $K \cap K^{g}=1$. Hence, $L:=\left\langle K, K^{g}\right\rangle=K \times K^{g} \leq X^{\prime}$, and $L$ is normal in $X$. Since $X^{\prime}$ is transitive on $\Delta, X^{\prime} / K$ induces a regular permutation group on $\Delta$, and in particular, $\left|X^{\prime} / K\right|=|\Delta|$. Let $Y=\left\langle X^{\prime}, g\right\rangle$. Then, $Y$ is normal in $X$ and transitive on $V$. Consider the quotient map $\mathcal{M}_{L}$ induced by $L$. Since $X^{\prime} / K$ is regular on $\Delta$, we have that $X^{\prime} / L$ is regular on $\Delta_{L}$. Hence, $Y / L$ is regular on $V_{L}=\Delta_{L} \cup \Delta_{L}^{\prime}$, and as $Y \triangleleft X, Y / L$ is normal in $X / L$. So $\mathcal{M}_{L}$ is a balanced Cayley map with respect to $Y / L$. Let $B$ be an $L$-orbit containing $\beta$. Then, $\alpha$ is adjacent to all points in $B$. It follows that $\mathcal{M}=\mathcal{M}_{L}\left[\bar{K}_{b}\right]$, where $b=|B|$, as in part (2)(ii).

Finally, if $|V|$ is odd, then part (1) occurs, and $\mathcal{M}$ is balanced. We note that the automorphism group of each cyclic group is abelian. If $\mathcal{M}$ is cyclic and $|V|$ is odd, it then follows that $\mathcal{M}$ is chiral.

Remark. The structure and embedding of rotary Cayley maps of abelian groups were investigated in a recent paper $[8$ in terms of the given base group. Proposition 5.5 tells us that for such a Cayley map, changing the base group may lead to a nicer description of the map than using the original base group.

\section{IMPRIMITIVE QUOTIENTS}

As shown in Proposition 5.1, normal quotients of Cayley graphs are not necessarily Cayley graphs. Imprimitive quotients of Cayley graphs are of course not necessarily Cayley graphs. We will see that 'most' imprimitive quotients of Cayley 
graphs are not Cayley graphs, but some imprimitive quotients of some important Cayley graphs are Cayley graphs.

6.1. Edge-transitive graphs and edge-transitive Cayley graphs. For a group $G$, a core-free subgroup $H<G$ and a subset $R \subset G$ such that $R=R^{-1}$ and $\langle H, R\rangle=G$, let

$$
\Sigma=\operatorname{Cos}(G, H, H R H),
$$

the coset graph of $G$ relative to $H$ and $R$. Then, $\Sigma$ is undirected, connected, and $G$-vertex-transitive. There are some natural Cayley graphs corresponding the triple $(G, H, R)$. Let $S \subset G$ be such that $R \subseteq S \subseteq H R H$, and let

$$
\Gamma=\operatorname{Cay}(G, S) \text {. }
$$

The vertex set $V \Gamma=G$ has a natural $G$-invariant partition:

$$
\mathcal{B}=[G: H]=V \Sigma, \text { the vertex set of } \Sigma .
$$

Lemma 6.1. Using notation defined above, we have

(i) $\Sigma \cong \Gamma_{\mathcal{B}}$, that is, $\Sigma$ is an imprimitive quotient of $\Gamma$;

(ii) if $S$ contains $R H$ or $H R$, then $\Gamma$ is connected;

(iii) if $S=\left\{h^{-1} g h \mid g \in R, h \in H\right\}$, then $\Sigma$ is a normal quotient of $\Gamma$.

In particular, each vertex-transitive graph is an imprimitive quotient of a Cayley graph.

Proof. The proof of this lemma is straightforward.

In the case where $\Sigma$ is arc transitive, we have

Lemma 6.2. Let $\Sigma=\operatorname{Cos}(G, H, H g H)$, where $g^{2} \in H$ and $\langle H, g\rangle=G$. Let $\Gamma=\operatorname{Cay}\left(G,\left\{g,\left(g^{-1}\right)\right\}\right)$ and $\left.\Delta=\operatorname{Cos}(G, K, K g K\rangle\right)$, where $\left\langle g^{2}\right\rangle \leq K \leq H \cap H^{g}$. Further, let $\mathcal{B}=[G: H], \mathcal{B}^{\prime}=[G: K], l=o(g)$ and $m=|G| / l$. Then,

$$
\Gamma \cong m \mathrm{C}_{l}, \quad \Delta \cong \frac{m l}{2} \mathrm{~K}_{2} \text {, and } \Sigma=\Gamma_{\mathcal{B}}=\Delta_{\mathcal{B}^{\prime}} .
$$

The lemma shows that each arc-transitive graph is an imprimitive quotient of an arc-transitive Cayley graph which consists of cycles. However, for some important classes of Cayley graphs, imprimitive quotients of Cayley graph are also Cayley graphs.

6.2. Some imprimitive quotients are Cayley graphs. The following simple lemma shows that some imprimitive quotients of Cayley graphs are still Cayley graphs.

Lemma 6.3. Let $\Gamma=\operatorname{Cay}(G, S)$, and let $\hat{G} \leq X \leq \operatorname{Aut} \Gamma$. Let $\mathcal{B}$ be an $X$-invariant partition of $V \Gamma$. Assume that there exists a subgroup $F<\hat{G}$ such that

$$
\hat{G}=F \hat{G}_{B} \text { and } F \cap \hat{G}_{B}=1 \text {, where } B \in \mathcal{B} \text {. }
$$

Then, the imprimitive quotient $\Gamma_{\mathcal{B}}$ is a Cayley graph of a group $H$, where $H$ is such that $\hat{H}=F$.

Proof. Observe that $\left|V \Gamma_{\mathcal{B}}\right|=|V \Gamma| /|B|$. Since $\hat{G}$ is regular on $V \Gamma, \hat{G}$ is transitive on $\mathcal{B}$, and since $\hat{G}=F \hat{G}_{B}$, it follows that $F$ is transitive on $\mathcal{B}$. Further, $|F|=$ $|\hat{G}| /\left|\hat{G}_{B}\right|=|V \Gamma| /|B|=\left|V \Gamma_{\mathcal{B}}\right|$. Thus, $F$ is regular on $V \Gamma_{\mathcal{B}}$, and $\Gamma_{\mathcal{B}}$ is a Cayley graph of a group $H$ such that $\hat{H}=F$. 
Here is an example. Let $G$ be an abelian group, and let $\Gamma=\operatorname{Cay}(G, S)$. Let $X$ be such that $\hat{G}<X \leq$ Aut $\Gamma$. If $X$ is primitive, then $X$ is classified in [21]. Assume that $X$ is imprimitive, and let $\mathcal{B}$ be an imprimitive block system of $X$ on $V$. Then, by [21, Lemma 3.1], both $\hat{G}_{B}$ and $\hat{G}^{\mathcal{B}}$ are regular, where $B \in \mathcal{B}$. Thus, the quotient graph $\Gamma_{\mathcal{B}}$ is a Cayley graph of $G / N$, where $N<G$ is such that $\hat{N}$ acts regularly on $B$.

Next, we present a family of quasiprimitive arc transitive Cayley graphs which have an imprimitive Cayley quotient.

Example 6.4. Let $X=\mathrm{S}_{p^{r}}$ where $p$ is a prime, acting on $\Omega=\left\{1,2, \ldots, p^{r}\right\}$. Then, $X$ has an elementary abelian subgroup $H \cong \mathbb{Z}_{p}^{r}$ which is regular on $\Omega$, and thus $X=G H$, where $G=X_{1} \cong \mathrm{S}_{p^{r}-1}$, such that $G \cap H=1$. Let $V=[X: H]$. Then $X$ acting on $V$ has a regular subgroup $G$. The action of $X$ acting on $V$ is imprimitive, and there exists an element $\rho \in \mathbf{N}_{X}(H)$ of order $p^{r}-1$ such that $L:=H .\langle\rho\rangle \cong \operatorname{AGL}\left(1, p^{r}\right)$. Now $\mathcal{B}=[X: L]$ is an $X$-invariant partition of $V$, and $X$ acting on $\mathcal{B}$ has a regular subgroup $F \cong \mathrm{S}_{p^{r}-2}$.

Take an involution $g \in \mathbf{N}_{X}(\langle\rho\rangle)$, and let

$$
\Sigma=\operatorname{Cos}(X, L, L g L) \text { and } \Gamma=\operatorname{Cos}(X, H, H g H) .
$$

Then

(i) $\Sigma$ is a Cayley graph of $F \cong \mathrm{S}_{p^{r}-2}$, and $\Gamma$ is a Cayley graph of $G \cong \mathrm{S}_{p^{r}-1}$;

(ii) $\Sigma$ is 2-arc transitive of valency $p^{r}$, and $\Gamma$ is an imprimitive cover of $\Sigma$.

It is obvious that $\mathrm{K}_{p^{r}-1}$ is a group-dual of $\Gamma$, and the triangle graph is a group-dual of $\Sigma$.

The next lemma of this section will be used in classifying quasiprimitive permutation groups containing a regular dihedral subgroup.

Lemma 6.5. Let $X$ be a transitive permutation group on $\Omega$ which contains a regular subgroup $G$. Let $\mathcal{B}$ be an $X$-invariant partition of $\Omega$ and $B \in \mathcal{B}$, and let $K$ be the kernel of $X$ acting on $\mathcal{B}$.

(1) Assume that $G$ is abelian. Then, $G \cap K$ is regular on $B$, and $G K / K \cong$ $G /(G \cap K)$ is regular on $\mathcal{B}$. In particular, $X$ acting on $\Omega$ is quasiprimitive if and only if $X$ is primitive.

(2) Assume that $G$ is a dihedral group. Then, either $G \cap K$ is regular on $B$ and $G K / K$ is regular on $\mathcal{B}$, or $G \cap K$ is semi-regular on $B$ with 2 orbits and $G K / K$ is transitive on $\mathcal{B}$ with point stabilizer isomorphic to $\mathbb{Z}_{2}$. In the latter case, $X / K$ has a cyclic regular subgroup. In particular, if $X$ is quasiprimitive on $\Omega$, then either $X$ is primitive on $\Omega$, or $X$ is a primitive permutation group of degree $|\Omega| / 2$ containing a cyclic regular subgroup.

Proof. Part (1) is proved in [21, Lemma 3.1]. We only need to prove part (2), and we thus assume that $G$ is dihedral.

Since $G$ is regular on $\Omega$, the normal subgroup $G \cap K$ is semiregular on $\Omega$ and $G$ acts transitively on $\mathcal{B}$. Now $G \cap K$ is the kernel of $G$ acting on $\mathcal{B}$, and hence $G /(G \cap K)$ is a transitive permutation group on $\mathcal{B}$. Since $G$ is dihedral, either $G \cap K$ is cyclic or $G \cap K$ is dihedral of index 2 in $G$. In the latter case, an orbit of $G \cap K$ in $\Omega$ has size $|\Omega| / 2$, that is, $|\mathcal{B}|=2$, and so $G \cap K$ is regular on $B$ and $G /(G \cap K) \cong \mathbb{Z}_{2}$ is regular on $\mathcal{B}$. Suppose now that $G \cap K$ is cyclic. Then, $G /(G \cap K)$ is a dihedral group. Since $G /(G \cap K)$ is a transitive permutation group on $\mathcal{B}$, it follows that 
either $G /(G \cap K)$ is regular on $\mathcal{B}$ or the point-stabilizer of $G /(G \cap K)$ has order 2. In the former, $G \cap K$ is regular on $B$; in the latter, $G \cap K$ is semi-regular on $B$ with two orbits.

Suppose that $X$ is quasiprimitive and imprimitive on $\Omega$, and suppose further that $X$ acts on $\mathcal{B}$ primitively. Since $X$ is quasiprimitive on $\Omega, X$ acts faithfully on $\mathcal{B}$, and so $G$ acts faithfully and transitively on $\mathcal{B}$. Thus, $G_{B}$ is core-free in $G$, where $B \in \mathcal{B}$. It follows as $G$ is a dihedral group that $G_{B} \cong \mathbb{Z}_{2}$, and so $|\mathcal{B}|=|\Omega| / 2$. Write $G=\langle a\rangle \rtimes\langle z\rangle \cong \mathrm{D}_{2 m}$, where $2 m=|\Omega|$. It follows that $\langle a\rangle$ is regular on $\mathcal{B}$. Thus, $X$ is a primitive permutation group on $\mathcal{B}$ and contains a cyclic regular subgroup $\langle a\rangle$.

The property stated in Lemma 6.5 enables us to extend the classification of the primitive permutation groups containing a regular abelian subgroup, given in [17] and [21, Corollary 1.2] independently, into the quasiprimitive case.

Corollary 6.6. A quasiprimitive permutation group $X$ of degree $n$ contains a cyclic regular subgroup $G$ if and only if one of the following holds:

(i) $\mathbb{Z}_{p} \cong G \leq X \leq \operatorname{AGL}(1, p)$, where $p$ is a prime;

(ii) $X \cong \mathrm{A}_{n}$ with $n$ odd, or $\mathrm{S}_{n}$, where $n \geq 4$;

(iii) $\operatorname{PGL}(d, q) \leq X \leq \operatorname{P\Gamma L}(d, q), G$ is a Singer subgroup of $X$, and $n=\frac{q^{d}-1}{q-1}$;

(iv) $X \cong \mathrm{P} \Gamma \mathrm{L}(2,8), G=\langle s \sigma\rangle \cong \mathbb{Z}_{9}$, where $\langle s\rangle$ is a Singer subgroup of $X$, and $\sigma \in X \backslash \operatorname{PSL}(2,8)$ such that $o(\sigma)=3$;

(v) $(X, n)=(\operatorname{PSL}(2,11), 11),\left(\mathrm{M}_{11}, 11\right)$, or $\left(\mathrm{M}_{23}, 23\right)$.

\section{Proof of Theorem 1.5}

In this section, we give a classification of quasiprimitive permutation groups that contain a dihedral regular subgroup; in particular, we prove Theorem 1.5 .

Let $X$ be a quasiprimitive permutation group on $\Omega$ that contains a regular dihedral subgroup.

Lemma 7.1. The group $X$ is 2-transitive.

Proof. Suppose that $X$ is imprimitive. Then, by Lemma 6.5 (2), there exists an $X$-invariant partition $\mathcal{B}$ of $\Omega$ such that $m=|\mathcal{B}|=n / 2$, and $X^{\mathcal{B}}$ is primitive and contains a cyclic regular subgroup. Thus, $X_{\omega}$ is a subgroup of $X_{B}$ of index 2, where $B \in \mathcal{B}$ and $\omega \in B$. Then, by Proposition 6.6, one of the following holds:

(a) $X=\mathrm{S}_{m}$, and $X_{B}=\mathrm{S}_{m-1}$;

(b) $X=\mathrm{M}_{11}, m=11$, and $X_{B}=\mathrm{M}_{10} \cong \mathrm{A}_{6} \cdot \mathbb{Z}_{2}$;

(c) $\operatorname{PGL}(d, q) \leq X \leq \operatorname{P\Gamma L}(d, q), m=\frac{q^{d}-1}{q-1}$, and $X_{B}=P_{1}$, a parabolic subgroup of $X$.

Suppose first that $X=\mathrm{S}_{m}$ and $X_{B}=\mathrm{S}_{m-1}$. Then, $X_{\omega}=\mathrm{A}_{m-1}$ is a subgroup of $\operatorname{soc}(X)=\mathrm{A}_{m}$, and $\left|\operatorname{soc}(X): X_{\omega}\right|=\left|\mathrm{A}_{m}: \mathrm{A}_{m-1}\right|=m=n / 2$. Thus, $\operatorname{soc}(X)$ is intransitive on $\Omega$, which is a contradiction since $X$ is quasiprimitive. Suppose now that $X=\mathrm{M}_{11}$ and $X_{B}=\mathrm{A}_{6} \cdot \mathbb{Z}_{2}$. Then, $X_{\omega}=\mathrm{A}_{6}$, and $G \cong \mathrm{D}_{22}$. Now $X_{B}=X_{\omega} \cdot \mathbb{Z}_{2}$ is a non-split extension and $|X| /\left|X_{B}\right|=11$. It follows that all involutions of $X_{B}$ belong to $X_{\omega}$, and each involution of $X$ is conjugate to some involution of $X_{\omega}$. In particular, an involution of $G$ is conjugate to some of $X_{\omega}$ and so fixes some points in $\Omega$, which is a contradiction. Thus, we conclude that

$$
\operatorname{PGL}(d, q) \leq X \leq \operatorname{P\Gamma L}(d, q), m=\frac{q^{d}-1}{q-1} \text {, and } X_{B} \cong P_{1} .
$$


Let $C=\langle a\rangle$ be the cyclic subgroup of $G$ of index 2 which acts regularly on $\mathcal{B}$. Then, $|C|=\frac{q^{d}-1}{q-1}, G \leq \mathbf{N}_{X}(C)$, and by Corollary [6.6, either $C$ is a Singer subgroup of $X$, or $X=\mathrm{P} \Gamma \mathrm{L}(2,8)$ and $C \cong \mathbb{Z}_{9}$. For the latter, there is no involution $x \in X$ such that $a^{x}=a^{-1}$; see the Atlas 9 . We conclude that $C$ is a Singer subgroup of $X$. It is then known that $\mathbf{N}_{X}(C)=\langle a\rangle .\langle\tau\rangle$ such that $\langle\tau\rangle \cong \mathbb{Z}_{d}$ and $a^{\tau}=a^{q}$; see 14, Theorem 7.3]. Since $G \leq\langle a\rangle .\langle\tau\rangle$ is dihedral, there exists some integer $i$ such that $a^{q^{i}}=a^{\tau^{i}}=a^{-1}$, so that $a^{q^{i}+1}=1$. Since $a$ has order $\frac{q^{d}-1}{q-1}$, we obtain that $q^{i}+1$ is divisible by $\frac{q^{d}-1}{q-1}$. It follows that $d=2$ and $i=1$. Writing $q=p^{e}$ for some prime $p$ and some $e \geq 1$, we have $X_{B} \cong \mathbb{Z}_{p}^{e} \cdot \mathbb{Z}_{q-1}$, and so $X_{\omega} \cong \mathbb{Z}_{p}^{e} \cdot \mathbb{Z}_{\frac{q-1}{2}}$; in particular, $q$ is odd. Now $X_{\omega}<\operatorname{soc}(X)=\operatorname{PSL}(2, q)$, and hence $\operatorname{soc}(X)$ is intransitive on $\Omega$, which is a contradiction since $X$ is quasiprimitive on $\Omega$.

Thus, $X$ is primitive on $\Omega$. By a result of Wielandt (see [36. Theorem 25.6]), $X$ is 2-transitive.

Hence, $X$ is affine or almost simple. The next two lemmas treat these two cases separately.

Lemma 7.2. Assume that $X$ is affine. Then $(G, X)$ is one of the pairs listed below:

\begin{tabular}{c|cccccc}
$G$ & $\mathrm{D}_{4}$ & $\mathrm{D}_{4}$ & $\mathrm{D}_{8}$ & $\mathrm{D}_{16}$ & $\mathrm{D}_{16}$ & $\mathrm{D}_{16}$ \\
\hline$X$ & $\mathrm{~A}_{4}$ & $\mathrm{~S}_{4}$ & $\operatorname{AGL}(3,2)$ & $2^{4} \rtimes \mathrm{A}_{6}$ & $2^{4} \rtimes \mathrm{A}_{7}$ & $\operatorname{AGL}(4,2)$
\end{tabular}

Proof. Now $\mathbb{Z}_{p}^{d} \triangleleft X \leq \operatorname{AGL}(d, p)$, where $p$ is a prime and $d \geq 1$, such that $|G|=p^{d}$. Since $G$ is dihedral, we have that $G$ is of even order, and so $p=2$. Further, $X$ contains an element of order $2^{d-1}$. It is known that $\operatorname{AGL}(d, 2)<\operatorname{GL}(d+1,2)$, and the largest order of a 2-element in $\operatorname{GL}(d+1,2)$ is the smallest value of $2^{e}$ which is greater than $d+1$. Hence, $2^{d-1} \leq 2^{e} \leq 2(d+1)$. It follows that $d \leq 4$. If $d=2$, then $X=\mathrm{A}_{4}$ or $\mathrm{S}_{4}$; if $d=3$, then $X=\operatorname{AGL}(3,2)$; if $d=4$, then $X=2^{4} \rtimes \mathrm{A}_{6}$, $2^{4} \rtimes \mathrm{A}_{7}$ or $\operatorname{AGL}(4,2)$.

Lemma 7.3. Assume that $X$ is almost simple. Then, $\left(X, G, X_{\omega}\right)$ is one of the following triples:

(i) $\left(\mathrm{S}_{2 m}, \mathrm{D}_{2 m}, \mathrm{~S}_{2 m-1}\right),\left(\mathrm{A}_{4 m}, \mathrm{D}_{4 m}, \mathrm{~A}_{4 m-1}\right)$;

(ii) $\left(\mathrm{M}_{12}, \mathrm{D}_{12}, \mathrm{M}_{11}\right),\left(\mathrm{M}_{22} \cdot \mathbb{Z}_{2}, \mathrm{D}_{22}, \operatorname{PSL}(3,4) \cdot \mathbb{Z}_{2}\right),\left(\mathrm{M}_{24}, \mathrm{D}_{24}, \mathrm{M}_{23}\right)$;

(iii) $X \unrhd \operatorname{PSL}\left(2, p^{e}\right), G=\mathrm{D}_{p^{e}+1}, X_{\omega} \unrhd \mathbb{Z}_{p}^{e} \rtimes \mathbb{Z}_{\frac{p^{e}-1}{2}}$, and $p^{e} \equiv 3(\bmod 4)$;

(iv) $X \unrhd \operatorname{PGL}\left(2, p^{e}\right), G=\mathrm{D}_{p^{e}+1}, X_{\omega} \unrhd \mathbb{Z}_{p}^{e} \rtimes \mathbb{Z}_{p^{e}-1}{ }^{2}$, and $p^{e} \equiv 1(\bmod 4)$.

Proof. Let $Y$ be a minimal subgroup of $X$ which contains $G$ and is primitive on $\Omega$. Then, again by Wielandt's theorem, $Y$ is 2-transitive, and so $\operatorname{soc}(Y)$ is primitive (see [4). Let $H=Y_{\omega}$, where $\omega \in \Omega$, and let $M$ be a maximal subgroup of $Y$ which contains $G$. Then, $Y=M H$. By the assumption on the minimality of $Y$, $M$ is imprimitive on $\Omega$, and we conclude that $\operatorname{soc}(Y) \not Z M$. Hence, $Y=M H$ is a maximal factorization of $Y$, which is given in [25]. Now $Y$ is 2-transitive on $[Y: H]$ of degree $n$, and $n=|G|$ is even, say $n=2 l$. Inspecting the classification given in [25, we conclude that $(\operatorname{soc}(Y), 2 l)$ is one of the following groups:

$$
\left(\mathrm{A}_{2 l}, 2 l\right),\left(\mathrm{M}_{12}, 12\right),\left(\mathrm{M}_{24}, 24\right),\left(\operatorname{PSL}(d, q), \frac{q^{d}-1}{q-1}\right) .
$$

Suppose first that $\operatorname{soc}(Y)=\mathrm{A}_{2 l}$. Then, $G=\mathrm{D}_{2 l}, Y=\mathrm{A}_{2 l}$ or $\mathrm{S}_{2 l}$, and $Y_{\omega}=\mathrm{A}_{2 l-1}$ or $\mathrm{S}_{2 l-1}$, respectively. If $Y=\mathrm{S}_{2 l}$, then obviously $X=Y$, as in part (i). Assume that $Y=\mathrm{A}_{2 l}$. If $l$ is odd, then any involution of $X$ fixes some points of $\Omega$, which 
is not possible, so that $l$ is even, say $2 l=4 m$. Thus, either $X=Y=\mathrm{A}_{4 m}$, or $X=Y . \mathbb{Z}_{2}=\mathrm{S}_{2 l}$, as in part (i).

We then suppose that $\operatorname{soc}(Y)=\mathrm{M}_{12}$ with $2 l=12$. By the Atlas $\left[9, Y=\mathrm{M}_{12}\right.$, and $H=Y_{\omega} \cong \mathrm{M}_{11}$. It is known that $Y=M H$, where $M \cong \operatorname{PSL}(2,11)$ (see 25. Table 6]), such that $M \cap H \cong \mathbb{Z}_{11} \cdot \mathbb{Z}_{5}$. Further, we have $M=G A$, where $A \cong \mathbb{Z}_{11} \cdot \mathbb{Z}_{5}$ and $G \cong \mathrm{D}_{12}$. It then follows that $Y=G H$, such that $G \cap H=1$. Now $Y \leq X \leq \mathrm{S}_{12}$, and it follows that either $X=Y=\mathrm{M}_{12}$, as in part (ii), or $X=\mathrm{A}_{12}$ or $\mathrm{S}_{12}$, as in part (i).

Next, suppose that $\operatorname{soc}(Y)=\mathrm{M}_{22}$ with $2 l=22$. By [25, Table6], we have that $Y \cong \mathrm{M}_{22} \cdot \mathbb{Z}_{2}$, and $Y=M H$ such that $H \cong \operatorname{PSL}(3,4) \cdot \mathbb{Z}_{2}$ and $M \cong \operatorname{PGL}(2,11)$. Further, $M=G A$ such that $A \cong \mathrm{A}_{5}$ and $G \cong \mathrm{D}_{22}$. It then follows that $Y=G H$ such that $G \cap H=1$. Now $Y \leq X \leq \mathrm{S}_{22}$, and it follows that either $X=Y=\mathrm{M}_{22} \cdot \mathbb{Z}_{2}$, as in part (ii), or $X=\mathrm{A}_{22}$ or $\mathrm{S}_{22}$, as in part (i).

Now we suppose that $\operatorname{soc}(Y)=\mathrm{M}_{24}$ with $2 l=24$. Then $Y=\operatorname{soc}(Y)=\mathrm{M}_{24}$ and $H=Y_{\omega}=\mathrm{M}_{23}$. It is known that $Y=M H$, where $M \cong \operatorname{PSL}(2,23)$; see [25, Table 6]. Then, $M \cap H \cong \mathbb{Z}_{23} \cdot \mathbb{Z}_{11}$, and $M=G A$, where $A \cong \mathbb{Z}_{23} \cdot \mathbb{Z}_{11}$ and $G \cong \mathrm{D}_{24}$. It then follows that $\operatorname{soc}(Y)=G H$, such that $G \cap H=1$. Now $Y \leq X \leq \mathrm{S}_{24}$, and it follows that either $X=Y=\mathrm{M}_{24}$, as in part (ii), or $X=\mathrm{A}_{24}$ or $\mathrm{S}_{24}$, as in part (i).

Suppose that $\operatorname{soc}(Y)=\operatorname{PSL}(d, q)$ and $2 l=\frac{q^{d}-1}{q-1}$ with $d \geq 3$. Then, the maximal factorization $Y=M H$ is given in [25, Table 1], such that $H=P_{1}$ is a parabolic subgroup. By [25, Table 1], either $d$ is even and $\operatorname{soc}(M) \cong \operatorname{PSp}(d, q)$, or $M=$ $\operatorname{GL}\left(a, q^{b}\right) \cdot \mathbb{Z}_{b}$ where $a b=d$. If $\operatorname{soc}(M)=\operatorname{PSp}(d, q)$, then it follows that $M$ is quasiprimitive, and by Lemma 7.1, $M$ is 2-transitive of degree $\frac{q^{d}-1}{q-1}$, which is not possible. Thus, we have that $M={ }^{\wedge} \operatorname{GL}\left(a, q^{b}\right) \cdot \mathbb{Z}_{b}$. Suppose that $a \neq 1$. Then $M / \mathbf{Z}(M)=\operatorname{PGL}\left(a, q^{b}\right) \cdot \mathbb{Z}_{b}$. It follows from Lemma 6.5 that $G \mathbf{Z}(M) / \mathbf{Z}(M) \cong$ $G /(G \cap \mathbf{Z}(M))$ has order $\frac{q^{a b}-1}{q^{b}-1}$, and hence $|G \cap \mathbf{Z}(M)|=\frac{q^{b}-1}{q-1}$. So $G$ is a central extension of $G \cap \mathbf{Z}(M)$ by $G /(G \cap \mathbf{Z}(M))$, which is a contradiction since $\frac{q^{b}-1}{q-1}=$ $|G \cap \mathbf{Z}(M)| \leq|\mathbf{Z}(G)| \leq 2$. Thus, $a=1$, and so $M=\operatorname{P\Gamma L}\left(1, q^{d}\right)$. However, it is easily shown that $\operatorname{P\Gamma L}\left(1, q^{d}\right)$ with $d \geq 3$ contains no dihedral subgroup of order $\frac{2\left(q^{d}-1\right)}{q-1}$, which is again a contradiction.

Finally suppose that $N:=\operatorname{soc}(Y)=\operatorname{PSL}(2, q)$ and $G=\mathrm{D}_{q+1}$. Then, $Y_{\omega}$ is a parabolic subgroup. Since $Y$ is primitive, $N$ is transitive and thus $\left|N_{\omega}\right|=q(q-1) / 2$ and $N_{\omega}=\mathbb{Z}_{p}^{e} \rtimes \mathbb{Z}_{\frac{p^{e}-1}{2}}$, where $q=p^{e}$. We note that all involutions of $N$ are conjugate, and $\operatorname{PGL}(2, q)$ has exactly two conjugacy classes of involutions, one inside $N$ and the other outside $N$. Now $G$ contains involutions which are not conjugate to the involutions of $N_{\omega}$ (if any). Thus, either $\left|N_{\omega}\right|$ is odd, or all involutions of $G$ lie in the same conjugacy class and all involutions of $N_{\omega}$ lie in the other. For the former, $\frac{p^{e}-1}{2}$ is odd, and so $p^{e} \equiv 3(\bmod 4)$. For the latter, $\frac{p^{e}-1}{2}$ is even, and there are involutions in $N_{\omega}$. Thus, the involutions of $G$ are not in $N$, and so $\frac{p^{e}+1}{2}$ is odd, that is, $p^{e} \equiv 1(\bmod 4)$ and $Y \geq \operatorname{PGL}(2, q)$. Since $Y \leq X \leq \mathrm{S}_{q+1}$, it follows that either $X=Y$, as in part (ii) or (iii), or $X=\mathrm{A}_{q+1}$ or $\mathrm{S}_{q+1}$, as in part (i).

Finally, we summarize the arguments for proving Theorem 1.5.

Proof of Theorem 1.5. Let $X$ be a quasiprimitive permutation group on $\Omega$ containing a dihedral regular subgroup $G$. By Lemma 7.1, we have that $X$ is 2-transitive on $\Omega$, and is affine or almost simple. If $X$ is affine, then by Lemma 7.2 , the pair 
$(X, G)$ satisfies Theorem 1.5(i). If $X$ is almost simple, then by Lemma 7.3, the pair $(X, G)$ satisfies one of parts (ii)-(v) of Theorem 1.5 .

\section{Proof of Theorem 1.6}

In the final section, we prove Theorem 1.6. The proof will consist of a number of lemmas.

Let $G$ be a finite simple group. By a result of [27, $G$ can be generated by two elements $\rho, g$, where $\rho$ has order $k>2$ and $g$ is an involution. Let $S=g^{\langle\rho\rangle}=$ $\left\{g, g^{\rho}, \ldots, g^{\rho^{k-1}}\right\}$, and let $N=\langle S\rangle$. Then, $\rho$ normalizes $N$, and hence $N$ is a normal subgroup of $G$. If $N<G$, then $\rho \notin N$, and hence $\langle g, \rho\rangle \leq\langle N, \rho\rangle\langle G$, which is a contradiction. So $\langle S\rangle=G$, and the Cayley graph $\operatorname{Cay}(G, S)$ is connected. Let $\rho^{*}$ be the inner automorphism of $G$ induced by $\rho$. Then, we have a rotary Cayley map $\mathcal{M}=\operatorname{Cay}\left(G, S, \rho^{*}\right)$. Thus, we have the first lemma, which tells us that all finite simple groups have rotary Cayley maps.

Lemma 8.1. Every finite simple group has a rotary Cayley map.

Moreover, applying the above argument with a result of 26, we have that 'most' finite simple groups have rotary Cayley maps of valency 3; see Proposition 5.1 and its Remark. However, the next lemma shows that not every simple group has a regular Cayley map and that not every simple group has a chiral Cayley map.

Lemma 8.2. (1) All rotary Cayley maps of the group $\mathrm{Sz}\left(2^{d}\right)$ are chiral.

(2) All rotary Cayley maps of the group $\operatorname{PSL}\left(2,2^{d}\right)$ are regular.

Proof. Let $G=\operatorname{Sz}\left(2^{d}\right)$ or $\operatorname{PSL}\left(2,2^{d}\right)$, and let $G=\langle\rho, g\rangle$, where $o(\rho=k \geq 3$ and $g$ is an involution. Let $S_{1}=\left\{g, g^{\rho}, \ldots, g^{\rho^{k-1}}\right\}$, and let $S_{2}=\left\{\rho, \rho^{-1}, \rho^{g},\left(\rho^{-1}\right)^{g}\right\}$. Then, $\mathcal{M}:=\operatorname{Cay}\left(G, S_{1}, \rho^{*}\right)$ is a rotary Cayley map, and $\Gamma:=\operatorname{Cay}\left(G, S_{2}\right)$ is edge-transitive. By [10, Theorem 1.1], $\hat{G} \triangleleft$ Aut $\Gamma$, that is, $\Gamma$ is a normal Cayley graph.

For $G=\mathrm{Sz}\left(2^{d}\right)$, by [10, Theorem 1.5], the graph $\Gamma$ is half-transitive, and thus by [28, Theorem 3.2], $\mathcal{M}$ is chiral. For $G=\operatorname{PSL}\left(2,2^{d}\right)$, by [10, Theorem 1.6], the graphs $\Gamma$ is arc-transitive, and thus by [28, Theorem 3.2], $\mathcal{M}$ is regular.

We are now ready to prove Theorem 1.6 .

Proof of Theorem 1.6. Let $G$ be a non-abelian simple group. Then, by Lemma 8.1 , $G$ has a normal rotary Cayley map. However, by Lemma 8.2(1), not every simple group has regular Cayley maps. On the other hand, by Lemma 8.2(2), not every simple group has chiral Cayley maps.

Now let $\mathcal{M}$ be a Cayley map of $G$ of valency $k$ with vertex set $V$, and let $X=$ Aut $\mathcal{M}$. Assume that $\mathcal{M}$ is rotary. Then $X=\hat{G} H$ is such that $\hat{G} \cap H=1$ and $H \cong \mathbb{Z}_{k}$ or $\mathrm{D}_{2 k}$. Since $G$ is simple, it is easily shown that either $\hat{G}$ is normal in $X$ or $\hat{G}$ is core-free in $X$. If $\hat{G}$ is normal in $X$, then $\mathcal{M}$ is a normal Cayley map of $\hat{G}$, as in part (i) of Theorem 1.6 .

We thus assume that $\hat{G}$ is core-free in $X$. Let $\Omega=[X: \hat{G}]$. Then $X$ acts faithfully on $\Omega$ and $H$ acts regularly on $\Omega$. Hence, $X$ is a permutation group which contains a cyclic or a dihedral regular subgroup $H$, and the point stabilizer $X_{\omega} \cong G$ is non-abelian simple.

Assume first that $X$ is quasiprimitive on $\Omega$. If $H$ is cyclic, then it follows from Corollary 6.6 that $(X, \hat{G})=\left(\operatorname{PSL}(2,11), \mathrm{A}_{5}\right),\left(\mathrm{M}_{23}, \mathrm{M}_{22}\right)$ or $\left(\mathrm{A}_{2 l+1}, \mathrm{~A}_{2 l}\right)$, as in 
part (ii). If $H$ is dihedral, then by Theorem 1.5, $(X, \hat{G})=\left(\mathrm{A}_{4 m}, \mathrm{~A}_{4 m-1}\right),\left(\mathrm{M}_{12}, \mathrm{M}_{11}\right)$ or $\left(\mathrm{M}_{24}, \mathrm{M}_{23}\right)$, as in part (iii).

Assume now that $X$ is not quasiprimitive on $\Omega$ in the following. Let $M$ be the socle of $X$, and let $Y=\langle M, \hat{G}\rangle=M \hat{G}$.

Case 1. Suppose that $\hat{G} \leq M$. Then $X=M H, M=\hat{G} M_{\mathbf{1}}$, and $\hat{G} \cap M_{\mathbf{1}}=1$. Further, $X / M=H M / M \cong H /(M \cap H)$, which is cyclic or dihedral. Since $\hat{G}$ is non-abelian simple, $\hat{G}$ is contained in one of the direct factors $T$ of $M$. As $M_{\mathbf{1}}$ is cyclic or dihedral, we conclude that $M$ is simple, and so $X$ is almost simple. Now $M$ has a transitive permutation representation on $[M: \hat{G}]$ which contains a cyclic or dihedral regular subgroup $M_{1}$. It follows from Corollary 6.6 and Theorem 1.5 that $(\hat{G}, M)$ is one of the following groups:

$$
\left(A_{n-1}, A_{n}\right),\left(A_{5}, \operatorname{PSL}(2,11)\right),\left(M_{22}, M_{23}\right),\left(M_{11}, M_{12}\right) \text { or }\left(M_{23}, M_{24}\right) \text {. }
$$

Then we have that either $X=M$, or $M=\mathrm{A}_{n}$ and $X=M . \mathbb{Z}_{2}$. If $(\hat{G}, M)=$ $\left(\mathrm{A}_{5}, \operatorname{PSL}(2,11)\right)$ or $\left(\mathrm{M}_{22}, \mathrm{M}_{23}\right)$, then $X=M$ and $\mathcal{M}$ is chiral, as in part (ii). If $(\hat{G}, M)=\left(\mathrm{A}_{n-1}, \mathrm{~A}_{n}\right)$ and $X=M$, then $n=2 m+1$ and $\mathcal{M}$ is chiral, as in part (ii). If $(\hat{G}, M)=\left(\mathrm{M}_{11}, \mathrm{M}_{12}\right)$ or $\left(\mathrm{M}_{23}, \mathrm{M}_{24}\right)$, then $\mathcal{M}$ is regular, as in part (iv). In the remaining case, we have that $(\hat{G}, M)=\left(\mathrm{A}_{n-1}, \mathrm{~A}_{n}\right)$, and either $X=M$ and $n=4 m$, or $X=M \cdot \mathbb{Z}_{2}$, which is isomorphic to $\mathrm{S}_{n}$ or $\mathrm{A}_{n} \times \mathbb{Z}_{2}$. For the former case, $\mathcal{M}$ is regular, as in part (iv). For the latter case, $\mathcal{M}$ is regular and $M=\mathrm{A}_{n}$ contains an element of order $n$, and hence $n=2 m+1$ is odd, also as in part (iv).

Case 2. Suppose that $\hat{G} \not \subset M$. Since $\hat{G}$ is simple and $M \cap \hat{G} \triangleleft \hat{G}$, we have that $M \cap \hat{G}=1$. Since $M$ is the socle of $X, \hat{G}$ does not centralise $M$. Hence, there exists a minimal normal subgroup $N$ of $X$ which is not centralised by $\hat{G}$. Now $N=T^{n}$, where $T$ is a simple group and $n \geq 1$. Let $Y=N \hat{G}$, and let $\Delta=[Y: \hat{G}]$. Then, $Y$ is transitive on $V$ and on $\Delta$, and $Y=\hat{G} Y_{1}$ such that $\hat{G} \cap Y_{1}=1$. Further, $Y_{1} \leq X_{1}$ is cyclic or dihedral, and $Y_{\mathbf{1}}$ is regular on $\Delta$. Since $N \cap \hat{G}=1$, we have $|N|=\left|Y_{\mathbf{1}}\right|$.

Suppose that $N$ is not a minimal normal subgroup of $Y$. Let $L$ be a maximal proper subgroup of $N$ which is normal in $Y$. Then $L=T^{l}$, where $1 \leq l<n$. Suppose that $L$ is transitive on $V$. Then $T^{n-l} \leq N_{1} \leq Y_{1}$ which is cyclic or dihedral, and hence $T^{n-l}=\mathbb{Z}_{p}$ with $p$ prime, or $\mathbb{Z}_{2}^{2}$. This is not possible since now $N$ is abelian, and so $N$ is regular on $V$. Thus, $L$ is intransitive on $V$. Let $\bar{N}=N / L$ and $\bar{Y}=Y / L$. Then, $\bar{N}=T^{n-l}, \bar{Y}=\bar{N} \rtimes G$, and $\bar{N}$ is the unique minimal normal subgroup of $\bar{Y}$. In particular, $\bar{Y}$ acts on $\Delta_{L}$ faithfully. Now $|L||N / L|=|N|=\left|Y_{1}\right|=\left|L \cap Y_{\mathbf{1}}\right|\left|Y_{\mathbf{1}} L / L\right|$. Further, $\left|L \cap Y_{\mathbf{1}}\right|$ divides $|L|$, and hence $|N / L|$ divides $\left|Y_{1} L / L\right|$. Since $N$ is transitive on $\Delta$, we have that $N / L$ is transitive on $\Delta_{L}$, and by Lemma 6.5, $Y_{1} L / L$ is transitive on $\Delta_{L}$ with point stabiliser of order at most 2. Thus, $\left|Y_{\mathbf{1}} L / L\right|=|N / L|$ or $2|N / L|$, and so $|L|=\left|Y_{\mathbf{1}} \cap L\right|$ or $2\left|Y_{\mathbf{1}} \cap L\right|$, that is, $Y_{1} \cap L$ is a subgroup of $L$ of index at most 2. Since $Y_{1}$ is cyclic or dihedral, we conclude that $L=\mathbb{Z}_{p}$ or $\mathbb{Z}_{2}^{2}$. Thus, $N=\mathbb{Z}_{p}^{r}$, where $p$ is a prime $p$ and some $r \geq 1$. Hence, either $Y_{\mathbf{1}} \cong \mathbb{Z}_{p^{r}}$, or $p=2$ and $Y_{\mathbf{1}} \cong \mathrm{D}_{2^{r}}$.

Consider the coset action of $\bar{Y}$ on $[\bar{Y}: \hat{G}]$. Since $\bar{N}$ is the unique minimal normal subgroup of $\bar{Y}$, we have that $\bar{Y}$ is quasiprimitive on $[\bar{Y}: \hat{G}]$ that contains a regular subgroup $\bar{Y}_{\mathbf{1}}$, which is cyclic or dihedral. By Lemma 6.5 and Theorem 1.5 , we conclude that $\bar{Y}$ is primitive on $[\bar{Y}: \hat{G}]$. By Theorem $\left[1.5, \bar{N}=\mathbb{Z}_{2}^{3}\right.$ or $\mathbb{Z}_{2}^{4}$, $\bar{Y}=\operatorname{AGL}(3,2), 2^{4} \rtimes \mathrm{A}_{6}, 2^{4} \rtimes \mathrm{A}_{7}$ or $\operatorname{AGL}(4,2)$, and $\hat{G}=\mathrm{GL}(3,2), \mathrm{A}_{6}, \mathrm{~A}_{7}$ or 
$\operatorname{GL}(4,2)$. Suppose that $\bar{N} ¥ N$. Then either $Y_{1} \cong \mathrm{D}_{16}$ or $\mathrm{D}_{32}$ and $\hat{G}=\mathrm{GL}(3,2)$, $\mathrm{A}_{6}$ or $\mathrm{A}_{7}$, or $Y_{\mathbf{1}} \cong \mathrm{D}_{32}$ or $\mathrm{D}_{64}$ and $\hat{G}=\mathrm{GL}(4,2)$. However, none of $\mathrm{GL}(3,2), \mathrm{A}_{6}$ and $A_{7}$ has elements of order 8 , and also $\operatorname{GL}(4,2) \cong A_{8}$ has no element of order 16. This contradiction leads to the proof that $N$ is a minimal normal subgroup. Thus, $Y$ is a quasiprimitive permutation group on $\Delta$ containing a regular cyclic or dihedral subgroup $Y_{\mathbf{1}}$. By Corollary 6.6 and Theorem 1.5. $Y_{\mathbf{1}}$ is dihedral of order at most 16. Since $\left|Y_{\mathbf{1}}\right|=|N|=p^{r}$, we have that $p=2, Y_{\mathbf{1}}=\mathrm{D}_{2^{r}}$, and $N=\mathbb{Z}_{2}^{r}$, where $r \leq 4$. In particular, this implies that $N=\mathbb{Z}_{2}^{r}$ is the unique minimal normal subgroup of $X$ which is not centralised by $G$.

Write $M=N \times O$. Then, $\hat{G}$ centralises $O$, and $Z=\langle M, \hat{G}\rangle=\langle O, N, \hat{G}\rangle=$ $(N \rtimes \hat{G}) \times O$. Thus, $Z_{\mathbf{1}} \cong \mathrm{D}_{2^{r}} \times O$. Since $Z_{1}$ is cyclic or dihedral, it follows that $O=1$ and $M=N$, that is, $N$ is the only minimal normal subgroup of $X$.

Let $C=\mathbf{C}_{X}(N)$, and let $Z=C \rtimes \hat{G}$. Then, $\left|Z_{\mathbf{1}}\right|=|C|=2^{r}|C / N|$ with $r \leq 4$, $C$ is a 2-group, and $N$ consists of all involutions of $C$. Assume that $r=4$, so that $\hat{G}=\operatorname{GL}(4,2) \cong \mathrm{A}_{8}$. Then, $\hat{G}$ has no element of order 16 , and it follows that $C$ contains element of order $2|C / N|$. Thus, $C / N$ is cyclic. Suppose that $C>N$. Then, it follows that $1 \neq \Phi(C) \cap N<N$, and so $\Phi(C) \cap N$ is a normal subgroup of $X$, which is a contradiction since $N$ is the unique minimal normal subgroup of $X$. Hence, $C=N$. Similarly, if $r \leq 3$, then $C=N$. Therefore, $Y=\bar{Y}=\operatorname{AGL}(3,2)$, $2^{4} \rtimes \mathrm{A}_{6}, 2^{4} \rtimes \mathrm{A}_{7}$ or $\operatorname{AGL}(4,2)$. Since $N$ is the unique minimal normal subgroup of $X$, we have that $X \leq N \rtimes \operatorname{Aut}(N) \cong \operatorname{AGL}(3,2)$ or $\operatorname{AGL}(4,2)$. It is now easily shown that $X=\operatorname{AGL}(3,2), 2^{4} \rtimes \mathrm{A}_{6}, 2^{4} \rtimes \mathrm{A}_{7}$ or $\operatorname{AGL}(4,2)$, and $\hat{G}=\mathrm{GL}(3,2), \mathrm{A}_{6}$, $\mathrm{A}_{7}$ or $\mathrm{A}_{8}$, as in part (iii) of Theorem 1.6 .

\section{REFERENCES}

[1] N. Biggs, Cayley maps and symmetrical maps, Proc. Camb. Phil. Soc. 72 (1972), 381-386. MR0302482 (46:1626)

[2] N. Biggs, Algebraic Graph Theory, Cambridge University Press, 2nd ed, 1993, New York. MR.1271140 (95h:05105)

[3] N. Biggs and A. T. White, Permutation groups and Combinatorial Structures, London Math. Soc. Lect. Notes 33 (Cambridge Univ. Press, Cambridge 1997). MR0540889 (80k:20005)

[4] P. J. Cameron, Permutation groups, London Mathematical Society Student Texts, 45. Cambridge University Press, Cambridge, 1999. x+220 pp. MR.1721031 (2001c:20008)

[5] Y. Q. Chen and C. H. Li, Relative difference sets fixed by inversion and Cayley graphs, J. Combin. Theory Ser. A 111 (2005), 165-173. MR2144861 (2006b:05059)

[6] M. Conder, On symmetries of Cayley graphs and the graphs underlying regular maps, in preparation.

[7] M. Conder and B. Everitt, Regular maps on non-orientable surfaces, Geom. Dedicata 56 (1995), no. 2, 209-219. MR1338960 (96g:05046)

[8] M. Conder, R. Jajcay and T. Tucker, Regular Cayley maps for finite abelian groups, preprint (2003).

[9] J. H. Conway, R. T. Curtis, S. P. Norton, R. A. Parker, and R. A. Wilson, Atlas of Finite Groups, Oxford Univ. Press, London/New York, 1985. MR0827219 (88g:20025)

[10] X. G. Fang, C. H. Li and M. Y. Xu, On edge-transitive Cayley graphs of valency four, European J. Combin. 25 (2004), 1107-1116. MR2083459

[11] W. Feit, Some consequences of the classification of finite simple groups, The Santa Cruz Conference on Finite Groups (Univ. California, Santa Cruz, Calif., 1979), 175-181, Proc. Sympos. Pure Math., 37, Amer. Math. Soc., Providence, R.I., 1980. MR0604576 (82c:20019)

[12] A. Gardiner, R. Nedela, J. Šráň and M. Škoviera, Characterisation of graphs which underlie regular maps on closed surfaces J. London Math. Soc. (2) 59 (1999), no. 1, 100-108. MR1688492(2000a:05104) 
[13] C. D. Godsil, On the full automorphism group of a graph, Combinatorica 1 (1981), 243-256. MR0637829 (83a:05066)

[14] B. Huppert, Finite Groups, (Springer-Verlag, Berlin, 1967).

[15] N. Itô, Über das Produkt von zwei abelschen Gruppen, Math. Z. 62 (1955), 400-401. MR0071426 (17:125b)

[16] R. Jajcay and J. Siran, A construction of vertex-transitive non-Cayley graphs, Australas. J. Combin. 10 (1994), 105-114. MR.1296944 (95f:05055)

[17] G. Jones, Cyclic regular subgroups of primitive permutation groups J. Group Theory $\mathbf{5}$ (2002), no. 4, 403-407. MR:1931365 (2003h:20004)

[18] C. H. Li, Finite CI-groups are soluble, Bull. London Math. Soc. 31 (1999), 419-423. MR1687493 (2000d:05056)

[19] C. H. Li, Finite s-arc transitive graphs of prime-power order, Bull. London Math. Soc. 33 (2001), 129-137. MR1815416 (2002d:05064)

[20] C. H. Li, On isomorphisms of finite Cayley graphs - a survey, Discrete Math. 256 (2002), 301-334. MR.1927074 (2003i:05067)

[21] C. H. Li, The finite primitive permutation groups containing an abelian regular subgroup, Proc. London Math. Soc. 87 (2003), 725-748. MR2005881(2004i:20003)

[22] C. H. Li, Finite $s$-arc transitive Cayley graphs and flag-transitive projective planes, Proc. Amer. Math. Soc. 133 (2004), 31-41. MR2085150 (2005g:20003)

[23] C. H. Li, Finite edge-transitive Cayley graphs and rotary Cayley maps, II, in preparation.

[24] C. H. Li, Z. P. Lu and D. Marušič, Finite primitive permutation groups with a small suborbit and their orbital graphs. J. Algebra 279 (2004), 749-770. MR2078940 (2005d:20003)

[25] M. Liebeck, C. E. Praeger and J. Saxl, The maximal factorizations of the finite simple groups and their automorphism groups, Mem. Amer. Math. Soc. 86 (1990), no. 432, iv+151 pp. MR.1016353 (90k:20048)

[26] M. Liebeck and A. Shalev, Classical groups, probabilistic methods, and the (2,3)-generation problem, Ann. Math. 144 (1996), 77-125. MR.1405944(97e:20106a)

[27] G. Malle, J. Saxl and T. Weigel, Generation of classical groups, Geom. Dedicata 49 (1994), 85-116. MR $1261575(95 \mathrm{c}: 20068)$

[28] D. Marušič and R. Nedela, Maps and half-transitive graphs of valency 4, European J. Combin. 19 (1998), 345-354. MR1621025 (99e:05069)

[29] P. Neumann, Helmut Wielandt on Permutation groups, in Helmut Wielandt: Mathmatical Works, Eds by B. Huppert and H. Schneider, pp. 3-20, (Berlin, New York, 1994).

[30] C. E. Praeger, The inclusion problem for finite primitive permutation groups, Proc. London Math. Soc. (3) 60 (1990), 68-88. MR.1023805 (90j:20009)

[31] C. E. Praeger, An O'Nan-Scott theorem for finite quasiprimitive permutation groups and an application to 2-arc transitive graphs, J. London. Math. Soc. 47 (1992), 227-239. MR1207945 (94f:05068)

[32] C. E. Praeger, Finite normal edge-transitive Cayley graphs, Bull. Austral. Math. Soc. 60 (1999), 207-220. MR:1711938 (2000j:05057)

[33] B. Richter, J. Širán̆, R. Jajcay, T. Tuker and M. Watkins, Cayley maps, J. Combin. Theory Ser. B 95 (2005), 189-245. MR 2171363

[34] M. Škoviera and J. Širáň, Regular maps from Cayley graphs. I. Balanced Cayley maps, Discrete Math. 109 (1992), 265-276. MR.1192388 (93k:05055)

[35] J. Širáň and M. Škoviera, Regular maps from Cayley graphs. II. Antibalanced Cayley maps, Discrete Math. 124 (1994), no. 1-3, 179-191. MR1258853 (94m:05069)

[36] H. Wielandt, Finite Permutation Groups, Academic Press, New York, 1964. MR 0183775 $(32: 1252)$

[37] M. Y. Xu, Automorphism groups and isomorphisms of Cayley digraphs, Discrete Math. 182 (1998), 309-320. MR1603719 (98i:05096)

[38] S. J. Xu, X. G. Fang, J. Wang and M. Y. Xu, On cubic s-arc transitive Cayley graphs of finite simple groups, Europ. J. Combin. 26 (2005), 133-143. MR2101041

School of Mathematics and Statistics, University of Western Australia, Crawley, 6009 WA, Australia - and - Department of Mathematics, Yunnan University, Kunming 650031, People's Republic of China

E-mail address: li@maths.uwa.edu.au 